\title{
Arbeitsmarktwirkungen der Mindestlohneinführung im Bauhauptgewerbe
}

\author{
Helmut Apel • Ronald Bachmann • Stefan Bender • Philipp vom Berge • \\ Michael Fertig • Hanna Frings • Marion König • Joachim Möller • Alfredo Paloyo • \\ Sandra Schaffner • Marcus Tamm • Matthias Umkehrer • Stefanie Wolter
}

Angenommen: 22. August 2012 / Online publiziert: 14. September 2012

(C) Institut für Arbeitsmarkt- und Berufsforschung 2012

Zusammenfassung Zum 1. Januar 1997 wurde im Bauhauptgewerbe ein Mindestlohn eingeführt, der bis heute besteht. Die vorliegende Studie fasst verschiedene Ergebnisse zu den Folgen dieser Mindestlohnregelung zusammen. In Ostdeutschland war die Betroffenheit durch den Mindestlohn zum Zeitpunkt der Einführung stärker ausgeprägt als in Westdeutschland. Zudem wird seit dessen Einführung ein hoher Anteil der gewerblichen Arbeitnehmer im ostdeutschen Bauhauptgewerbe nahe dieser Untergrenze entlohnt. In Westdeutschland ist dies nicht der Fall. Für die Mindestlohneinführung zeigen sich positive Auswirkungen auf das Lohnwachstum in beiden Landesteilen. Nur sehr geringe

Für die in diesem Beitrag publizierten Ergebnisse und Schlussfolgerungen zeichnen ausschließlich die Autorinnen und Autoren verantwortlich. Sie geben nicht notwendigerweise die Position des Bundesministerium für Arbeit und Soziales wieder.

Dr. H. Apel · Dr. M. Fertig

Institut für Sozialforschung und Gesellschaftspolitik (ISG),

Barbarossaplatz 2, 50674 Köln, Deutschland

Dr. H. Apel

e-mail: apel@isg-institut.de

Dr. M. Fertig

e-mail: fertig@isg-institut.de

Dr. R. Bachmann · H. Frings · Dr. A. Paloyo · Dr. S. Schaffner .

Dr. M. Tamm

Rheinisch-Westfälisches Institut für Wirtschaftsforschung (RWI),

Hohenzollernstraße 1-3, 45128 Essen, Deutschland

Dr. R. Bachmann

e-mail: Ronald.Bachmann@rwi-essen.de

H. Frings

e-mail: Hanna.Frings@ @rwi-essen.de

Dr. A. Paloyo

e-mail: Alfredo.Paloyo@rwi-essen.de

Dr. S. Schaffner

e-mail: Sandra.Schaffner@rwi-essen.de oder keine Folgen lassen sich für die Beschäftigung inländischer Arbeitnehmer nachweisen. Auswirkungen auf ausländische Betriebe und deren Beschäftigte sowie Selbständige können kausalanalytisch nicht untersucht werden. Aufgrund der Besonderheiten des Bauhauptgewerbes ist bei der Übertragbarkeit der Ergebnisse auf andere Wirtschaftszweige bzw. Gesamtdeutschland allerdings Vorsicht geboten.

\section{Labour Market Effects of the Minimum Wage Introduction in the Main Construction Sector}

Abstract On January 1st, 1997, minimum wages were introduced in the German main construction sector. We examine the effects of this wage regulation on various aspects of the labour market of this sector, particularly the effects on wages and employment. In East Germany, at the time of its introduction, the minimum wage was more strongly binding than in West Germany. Furthermore, the wages of

\footnotetext{
Dr. M. Tamm

e-mail: Marcus.Tamm@rwi-essen.de

S. Bender · P. vom Berge · M. König ( $₫)$.

Prof. Dr. Dr. h.c. J. Möller · M. Umkehrer · S. Wolter Institut für Arbeitsmarkt und Berufsforschung (IAB), Regensburger Straße 104, 90478 Nürnberg, Deutschland e-mail: Marion.Koenig@iab.de

S. Bender

e-mail: Stefan.Bender@iab.de

P. vom Berge

e-mail: Philipp.Berge@iab.de

Prof. Dr. Dr. h.c. J. Möller

e-mail: Joachim.Moeller@iab.de

M. Umkehrer

e-mail: Matthias.Umkehrer@iab.de

S. Wolter

e-mail: Stefanie.Wolter@iab.de
} 
a large fraction of the construction workforce in East Germany are close to the minimum wage, which is not the case in West Germany. Our results indicate that the wage floor significantly increased wage growth but had a negligible impact on employment of domestic workers. Because of data restrictions, a causal analysis of the effects on foreign establishments and their employees or on the self-employed is not possible. We caution against drawing conclusions from the results of our study for other industries given the specific characteristics of the main construction sector in Germany.

Keywords Minimum wages - Labour market policy · Employment · Transitions · Construction industry

JEL Classification J31 $\cdot$ J38

\section{Einleitung}

Dieser Artikel fasst die Ergebnisse der vom Bundesministerium für Arbeit und Soziales (BMAS) in Auftrag gegebenen Studie zur „Evaluation bestehender gesetzlicher Mindestlohnregelungen - Branche: Bauhauptgewerbe“ zusammen. Diese wurde von einem Forschungskonsortium aus IAB Nürnberg, RWI Essen und ISG Köln realisiert (IAB/RWI/ISG 2011).

Das Bauhauptgewerbe stellt die erste Branche in Deutschland dar, in der ein Mindestlohn eingeführt wurde. Der Mindestlohn wurde von Gewerkschaften und Arbeitgebern ausgehandelt, für allgemeinverbindlich erklärt und trat zum 01. Januar 1997 in Kraft. ${ }^{1}$ Er ist für alle im räumlichen Geltungsbereich des Bundesrahmentarifvertrags für das Baugewerbe (BRTV) beschäftigten Arbeitnehmer bindend, und somit auch für jene, die bei einem ausländischen Arbeitgeber angestellt sind und nach Deutschland entsandt werden. Nicht in den Geltungsbereich fallen Auszubildende und Angestellte. Aufgrund der Größe des Bauhauptgewerbes und der Zahl der betroffenen Arbeitnehmer, Arbeitgeber und Auftraggeber sind potenzielle Effekte auf diese Branche auch für die Volkswirtschaft als Ganzes von großer Wichtigkeit.

Tabelle 1 zeigt die Höhe der Mindestlöhne im Bauhauptgewerbe in ihrer zeitlichen Entwicklung. Grundsätzlich wird zwischen Beschäftigung in Ost- und Westdeutschland sowie zwei Mindestlohnstufen unterschieden. Die Mindestlohnstufe I gilt für einfache Bau- und Montagetätigkeiten, für die keine Qualifikation vorausgesetzt wird. Für Fachkräfte ist der Mindestlohn II zu zahlen, der 2003 eingeführt - und in Ostdeutschland 2009 wieder abgeschafft - wurde. Seit der Einführung gab es vor allem moderate Erhöhungen des Mindestlohns, manchmal sogar Absenkungen (1997, 2005). Eine Ausnahme bildet die Änderung im Jahr 1999, bei der der

\footnotetext{
${ }^{1}$ Bekanntgabe am 12. November 1996 im Bundesanzeiger vom 16. November 1996, Nr. 215, S. 12102.
}

Mindestlohn in West- und Ostdeutschland deutlich angehoben wurde.

Vergleicht man das Bauhauptgewerbe mit anderen Branchen, so kommt man zu dem Schluss, dass sich die Strukturen deutlich von den meisten anderen Branchen der deutschen Wirtschaft unterscheiden. Für eine sinnvolle Einordnung der Ergebnisse, gerade in Hinblick auf die Einführung eines allgemeinen gesetzlichen Mindestlohns, ist ein Grundverständnis dieser Strukturen sowie der Umstände der Mindestlohneinführung daher unabdingbar. Diese werden im nachfolgenden Abschnitt erörtert. Die präsentierten Ergebnisse stützen sich auf eine einzigartige Datenbasis, die in Abschn. 3 erläutert wird. Bei den Analysen kommen sowohl quantitative als auch qualitative Evaluationsmethoden zum Einsatz, siehe Abschn. 4. Im Fokus der empirischen Analysen stehen in Abschn. 5 Wirkungen auf Löhne und deren Verteilung sowie in Abschn. 6 Beschäftigungswirkungen. Des Weiteren werden in Abschn. 7 Effekte auf die Wettbewerbsfähigkeit der Bauwirtschaft auf Produkt- und Arbeitsmärkten, sowie der Einfluss auf Aspekte der Arbeitsplatzqualität und -sicherheit untersucht. Neben kausaler expost Evaluation fließen auch Ergebnisse aus einer eigens für das Projekt durchgeführten Beschäftigtenbefragung und Informationen aus leitfadengestützten Experteninterviews ein, deren Ergebnisse in den Abschn. 8 und 9 präsentiert werden.

\section{Rahmenbedingungen der Mindestlohneinführung}

Das Bauhauptgewerbe hebt sich von anderen Wirtschaftzweigen in vielerlei Hinsicht ab. Zum einen unterliegt der Bauarbeitsmarkt saisonal und konjunkturell bedingten Nachfrageschwankungen. Zum anderen hat die Nichtlagerfähigkeit der Endprodukte, welche in der Regel Einzelanfertigungen für wechselnde Auftraggeber sind, hohe Produktionsschwankungen und damit Arbeitsplatzunsicherheit, Arbeitskräftefluktuation und Investitionsrisiken zur Folge (Unger und van Waarden 1993; Kofner 1998; Bosch et al. 2011). Relativ zu anderen Sektoren würde dies ohne zusätzliche Regelungen für den Bauarbeitsmarkt ein erhöhtes Zugangsrisiko in (saisonale) Arbeitslosigkeit und reduzierte Urlaubsansprüche sowie geringe Rentenansprüche durch fehlende Beitragszeiten bedeuten (Worthmann und Zühlke-Robinet 2003: 93). Zusätzlich bedingt die schwankende Bautätigkeit und die überwiegende Beschäftigung in kleinen Betrieben eine Erschwerung des Aufbaus betrieblicher Arbeitsmärkte, wie Bosch et al. (2002: 110) anmerken.

Weitere Besonderheiten ergeben sich durch die spezielle Preisbildung im Rahmen eines Ausschreibungsprozesses, welcher den (Groß-)Kunden eine nicht unerhebliche Preissetzungsmacht verleiht und dieses System der Auftragsvergabe somit als Nachfragemonopol (Monopson) erscheinen 
Tab. 1 Mindestlöhne im Bauhauptgewerbe Januar 1997 bis Januar 2012 West- und Ostdeutschland, in Euro

\begin{tabular}{|c|c|c|c|c|}
\hline \multirow[t]{2}{*}{ Geltungsdauer } & \multicolumn{2}{|l|}{ West } & \multicolumn{2}{|l|}{ Ost } \\
\hline & Mindestlohn I & Mindestlohn II für Fachkräfte & Mindestlohn I & Mindestlohn II für Fachkräfte \\
\hline \multirow[t]{2}{*}{$1 / 97-8 / 97$} & 8,69 & & 8,00 & \\
\hline & $(17,00)^{\mathrm{a}}$ & & $(15,64)^{\mathrm{a}}$ & \\
\hline \multirow[t]{2}{*}{ 9/97-8/99 } & 8,18 & & 7,74 & \\
\hline & $(16,00)^{\mathrm{a}}$ & & $(15,14)^{\mathrm{a}}$ & \\
\hline \multirow[t]{2}{*}{$9 / 99-8 / 00$} & 9,46 & & 8,32 & \\
\hline & $(18,50)^{\mathrm{a}}$ & & $(16,28)^{\mathrm{a}}$ & \\
\hline $9 / 00-8 / 01$ & 9,65 & & 8,49 & \\
\hline $9 / 01-8 / 02$ & 9,80 & & 8,63 & \\
\hline $9 / 02-8 / 03$ & 10,12 & & 8,75 & \\
\hline $9 / 03-10 / 03$ & 10,36 & 12,47 & 8,95 & 10,01 \\
\hline $11 / 03-8 / 04$ & 10,36 & 12,47 & 8,95 & 9,65 \\
\hline $9 / 04-08 / 05$ & 10,36 & 12,47 & 8,95 & 10,01 \\
\hline $9 / 05-8 / 06$ & 10,20 & 12,30 & 8,80 & 9,80 \\
\hline $9 / 06-8 / 07$ & 10,30 & 12,40 & 8,90 & 9,80 \\
\hline $9 / 07-8 / 08$ & 10,40 & 12,50 & 9,00 & 9,80 \\
\hline \multirow[t]{2}{*}{ 9/08-8/09 } & 10,70 & 12,85 & 9,00 & 9,80 \\
\hline & & & $(10,70)^{b}$ & $(12,70)^{\mathrm{b}}$ \\
\hline \multirow[t]{2}{*}{$9 / 09-8 / 10$} & 10,80 & 12,90 & 9,25 & - $^{\mathrm{c}}$ \\
\hline & & & $(10,80)^{b}$ & $(12,75)^{b}$ \\
\hline \multirow[t]{2}{*}{$9 / 10-6 / 11$} & 10,90 & 12,95 & 9,50 & $\mathrm{c}^{\mathrm{c}}$ \\
\hline & & & $(10,90)^{b}$ & $(12,75)^{\mathrm{b}}$ \\
\hline \multirow[t]{2}{*}{$7 / 11-12 / 11$} & 11,00 & 13,00 & 9,75 & $-^{c}$ \\
\hline & & & $(11,00)^{b}$ & $(12,85)^{b}$ \\
\hline \multirow[t]{2}{*}{ ab $1 / 12$} & 11,05 & 13,40 & 10,00 & $-^{c}$ \\
\hline & & & $(11,05)^{b}$ & $(13,25)^{b}$ \\
\hline
\end{tabular}

a) in DM; ${ }^{\text {b) }}$ gesonderte Mindestlöhne für das Land Berlin; ${ }^{\text {c) }}$ abgeschafft

Anmerkung: Bis August 2003 galt der Mindestlohn I für alle Baubeschäftigte, ab September 2003 gilt für Fachkräfte der höhere Mindestlohn II Quelle: Bosch und Zühlke-Robinet (2000), Platzer und Röschert (2005), verschiedene Tarifverträge Mindestlohn, SOKA-BAU; eigene Zusammenstellung und Umrechnung

lässt. Problematisch ist insbesondere, dass bei starkem Wettbewerb auf dem Baumarkt auch nicht kostendeckende Angebote abgegeben werden (Bauer 1995: 588). Darüber hinaus besteht eine relativ starke Abhängigkeit des Bauhauptgewerbes von der staatlichen Baunachfrage, deren Unstetigkeit aufgrund sich ändernder politischer Zielvorstellungen und Abhängigkeit von der Finanzlage zu Anpassungsproblemen in der Bauwirtschaft führen kann (Schneider et al. 1982: 58). Hingegen ist die Kapitalintensität der Bautätigkeit relativ gering (Hochstadt 2002: 44). ${ }^{2}$ Somit ist bei einer Unternehmensgründung verhältnismäßig wenig Kapital er-

\footnotetext{
${ }^{2}$ Laut den Daten der Volkswirtschaftlichen Gesamtrechnung des Statistischen Bundesamtes (Destatis 2011) lag das Verhältnis der Arbeitnehmerentgelte zur Bruttowertschöpfung zwischen 1991 und $2010 \mathrm{im}$ Baugewerbe im Durchschnitt sieben Prozentpunkte höher als in der Gesamtwirtschaft.
}

forderlich, was einen starken Wettbewerb nach sich zieht. Außerdem existieren größtenteils tarifvertraglich vereinbarte bauspezifische Regulierungssysteme, welche die soziale Absicherung der Beschäftigten, eine Verstetigung der Baubeschäftigung sowie die Steigerung der Attraktivität und Wettbewerbsfähigkeit der Bauwirtschaft gewährleisten sollen. Hierzu zählen insbesondere die Einrichtung der Sozialkassen $^{3}$ für überbetriebliche Zusatzleistungen, betriebliche Ausgleichskonten, spezielle Regelungen für die Winterzeit oder aber auch der Mindestlohn.

Wesentliche Charakteristika der Arbeitsbeziehungen im Bauhauptgewerbe sind nach Meinung von Bosch und

\footnotetext{
${ }^{3}$ Die Sozialkasse des Baugewerbes ist die SOKA-BAU, welche sich in zwei rechtlich selbständige Einrichtungen, der Urlaubs- und Lohnausgleichskasse (ULAK) sowie der Zusatzversorgungskasse (ZVK) untergliedert.
} 
Zühlke-Robinet (2000: 118) bundesweit geltende Tarifverträge zur Sicherung von einheitlichen Mindeststandards auf den Baustellen, auf denen Arbeitskräfte aus überregional tätigen Baubetrieben miteinander kooperieren. Der für allgemeinverbindlich erklärte Bundesrahmentarifvertrag für das Baugewerbe (BRTV) ist einer der wichtigsten Tarifverträge für die gewerblichen Arbeitnehmer im Bauhauptgewerbe. Durch ihn werden die Rahmenbedingungen für eine gewerbliche Beschäftigung geregelt, indem unter anderem Arbeitszeitregelungen, Lohngruppen sowie verschiedene Lohnbestandteile definiert werden. Die zwischen den Tarifparteien ausgehandelten stündlichen Vergütungen der Lohngruppen 1 und 2 fungieren als Mindestlohn I bzw. Mindestlohn II und sind im Tarifvertrag Mindestlohn festgelegt. Aufgrund der Allgemeinverbindlicherklärung des Mindestlohntarifvertrags ${ }^{4}$ gelten diese auch für nichtorganisierte Unternehmen.

Der Tarifbindungsgrad ist sowohl in West- als auch in Ostdeutschland deutlich höher als im Durchschnitt der Gesamtwirtschaft. ${ }^{5}$ In Ostdeutschland ist er traditionell aber relativ gering. Auch hinsichtlich der Tarifpolitik bestehen grundlegende Unterschiede zwischen den Bundesländern: Während die westdeutschen Verbände nach Expertenaussagen tendenziell auf die Angleichung der Tariflohnhöhen zwischen Ost- und Westdeutschland hinarbeiten, sind die Ansichten der ostdeutschen Verbände eher durch den intensiven Preiswettbewerb geprägt.

Das Baugewerbe ${ }^{6}$ zählt nach wie vor zu den bedeutendsten Wirtschaftszweigen in Deutschland. 2004 wurden etwa elf Prozent des gesamtwirtschaftlichen Produktionswertes durch die Wertschöpfungskette Bau generiert, wobei ein Anteil von etwa 40 Prozent auf das Baugewerbe selbst entfiel. Somit nimmt der Bausektor einen Anteil von ca. fünf Prozent am gesamtwirtschaftlichen Produktionswert und ca. vier Prozent an der Bruttowertschöpfung ein (IW Consult 2008; Destatis 2011). Dies ist allerdings vor dem Hintergrund stetig fallender Wertschöpfungsanteile nach dem Rückgang des großen Baubedarfs in den neuen Bundesländern im Zuge der Wiedervereinigung zu sehen. Nachdem der Bauboom 1995 seinen Höhepunkt erreicht hatte, erlebte das Baugewerbe einen bis 2005 andauernden konjunkturellen Abschwung.

\footnotetext{
${ }^{4}$ Bis 1999 wurde der Mindestlohntarifvertrag über das Tarifvertragsgesetz für allgemeinverbindlich erklärt. Im Zuge einer Änderung des Arbeitnehmer-Entsendegesetzes (AEntG) wurde dies auch über Rechtsverordnung des Bundesministeriums für Arbeit und Soziales möglich.

${ }^{5}$ Siehe hierzu Abb. 4.2 in IAB/RWI/ISG (2011).

${ }^{6}$ Dieser Bericht bezieht sich, wenn möglich, auf das Bauhauptgewerbe. In wenigen Fällen werden jedoch Zahlen für das Baugewerbe herangezogen, was das Ausbaugewerbe mit einschließt. Detaillierte Informationen zur Abgrenzung des Bauhauptgewerbes und der Mindestlohnbetroffenheit finden sich in IAB/RWI/ISG (2011: 44 ff).
}

Neben der Bruttowertschöpfung sanken auch Auftragseingänge, Umsatz und Investitionen und die Beschäftigung halbierte sich bei steigenden Material- und Lohnkosten nahezu. Die Preise für Endprodukte haben sich zwischen 1995 und 2005 allerdings kaum verändert, was den starken Preiswettbewerb auf dem Produktmarkt erahnen lässt. Auch die Zahl der Betriebe reduzierte sich kaum und ist in Ostdeutschland sogar gestiegen, was wiederum auf eine Veränderung der Betriebsgrößenstruktur hin zu einigen Großunternehmen und einer Vielzahl von Klein- bis Kleinstbetrieben zurückzuführen ist.

Aufgrund der Zunahme von Entsendungen zu Beginn der 1990er Jahre wurde insbesondere die Konkurrenz durch von ausländischen Firmen zur Erfüllung kurzfristiger Dienstleistungen nach Deutschland entsandte Arbeitnehmer als problematisch angesehen. Hierbei sind Entsendungen aus EU-Mitgliedstaaten, die im Rahmen der uneingeschränkten Dienstleistungsfreiheit erfolgen, und Entsendungen aus mittel- und osteuropäischen (MOE-) Staaten sowie der Türkei, die auf bilateralen Regierungsvereinbarungen beruhen, zu unterscheiden. Für Erstere gilt Genehmigungs- und Kontingentfreiheit. Da der EG-Vertrag mit Einführung der Dienstleistungsfreiheit keinerlei arbeits- und sozialrechtliche Bedingungen festgelegt hat, die bei Entsendungen einzuhalten sind, konnten Vorteile aufgrund eines niedrigen Lohnniveaus und geringen Arbeits- und Sozialstandards im Entsendeland ausgenutzt werden. Entsendungen aus MOEStaaten werden über kontingentierte Werkverträge geregelt, mit dem Ziel diese Staaten an Westeuropa heranzuführen, die wirtschaftlichen Beziehungen zu stärken und den Wanderungsdruck aus den MOE-Staaten abzubauen (Worthmann 1997). ${ }^{7}$ Zwar orientiert sich die Entlohnung am deutschen Netto-Tariflohn (einschließlich Auslösung und Urlaubsgeld), allerdings richten sich Sozialversicherung und Krankenschutz nach den Regelungen des Entsendelandes (Bosch und Zühlke-Robinet 2000).

Insgesamt kann davon ausgegangen werden, dass durch den Einsatz ausländischer Subunternehmer Lohnkostenvorteile realisiert werden konnten. ${ }^{8}$ Die Einführung eines Mindestlohns in Verbindung mit dem ArbeitnehmerEntsendegesetz (AEntG) sollte die Verbindlichkeit deutscher Mindestarbeitsbedingungen erreichen, tatsächliche oder vermeintliche Wettbewerbsverzerrungen durch teilweise erhebliche Lohnkostenvorteile von ausländischen Subunternehmern im Bauhauptgewerbe eindämmen und dem starken Lohndruck entgegenwirken. Das AEntG verpflichtet al-

\footnotetext{
${ }^{7}$ Deutsche Werkvertragsabkommen bestanden mit Bulgarien, BosnienHerzegowina, Lettland, Kroatien, Mazedonien, Polen, Rumänien, Serbien-Montenegro, Slowakei, Slowenien, Tschechien, Türkei und Ungarn. Die Werksvertragspflicht für Lettland, Polen, Slowakei, Slowenien, Tschechien und Ungarn ist zum 01. Mai 2011 entfallen.

${ }^{8}$ Cremers (2011) schätzt die Kostenvorteile, die auf Unterschieden in den Sozialversicherungskosten beruhen, auf 25 bis 30 Prozent.
} 
le ausländischen entsendenden Betriebe den nach Deutschland entsandten Beschäftigten die Arbeitsbedingungen des Gastlandes zu garantieren und die für allgemeinverbindlich erklärten Tarifverträge in Bezug auf Mindestlohn- und Urlaubsregelungen anzuwenden. Allerdings war dieses Gesetz keineswegs unumstritten. Nach harten Verhandlungen wurde der Tarifvertrag zur Regelung eines Mindestlohns vom 2. September 1996 am 12. November 1996 für allgemeinverbindlich erklärt und trat zum 1. Januar 1997 in Kraft. ${ }^{9}$ Die Wirkungen dieses Mindestlohns werden nun im Folgenden dargestellt.

\section{Datenbasis}

Die zentrale Datenbasis für nachfolgende Analysen bildet der Administrative Linked-Employer-Employee-Datensatz für das Bauhauptgewerbe (ALEED-Bau). Er basiert auf einer Vollerhebung aller Betriebe und Beschäftigten des Bauhauptgewerbes für den Zeitraum von Januar 1993 bis Dezember 2009 und wurde eigens für das Forschungsprojekt erstellt.

Der Datensatz setzt sich einerseits aus administrativen Datenquellen der Bundesagentur für Arbeit zusammen, die es ermöglichen, das Entgelt, die Erwerbshistorie und Charakteristika individueller Arbeitnehmer sowie verschiedene Merkmale der beschäftigenden Betriebe über den Zeitraum 1993 bis 2009 zu verfolgen. Hierbei wurde auf die Integrierten Erwerbsbiographien (IEB) und das Betriebs-Historik-Panel (BHP) zurückgegriffen. Diese Datenquellen werden durch weitere Betriebsinformationen aus dem IAB-Betriebspanel sowie durch imputierte Arbeitszeitinformationen für Arbeitnehmer aus dem Mikrozensus, die für die Berechnung der Stundenlöhne benötigt werden, angereichert. Des Weiteren enthält der ALEEDBau die Informationen einer Beschäftigtenbefragung, die zu Jahresbeginn 2011 innerhalb einer Auswahl von Betriebspanelbetrieben des Bauhauptgewerbes durchgeführt wurde.

Anhand der IEB können komplette Erwerbsbiografien mit tagesgenauen Angaben zur Beschäftigung nachvollzogen werden (vgl. Oberschachtsiek et al. 2009 für eine detaillierte Beschreibung). Nachteil der administrativen Daten ist, dass keine tatsächlich geleisteten Arbeitszeiten erfasst werden. Diese werden für Beschäftigte im Bauhauptgewerbe und den Kontrollbranchen mithilfe einer Regression aus dem Mikrozensus imputiert. Da außerdem die überwältigende Mehrheit der gewerblich Beschäftigten im Bauhauptgewerbe männliche vollzeitbeschäftigte Arbeiter sind, wird

\footnotetext{
${ }^{9}$ Die Rechtsverbindlichkeit der Mindestlohnregelungen folgte dabei aus $\S 1$ Abs. 1 AEntG in Verbindung mit der Allgemeinverbindlicherklärung nach $§ 5 \mathrm{TVG}$.
}

der Datensatz auf diese Gruppe eingegrenzt. Nebentätigkeit, Heimarbeit und Teilzeitbeschäftigung bleiben daher unberücksichtigt, ebenso wie Meister, Angestellte und Auszubildende. Des Weiteren erfolgen alle Untersuchungen separat für die neuen und die alten Bundesländer. Berlin bleibt aufgrund seines geographischen Sonderstatus unberücksichtigt.

Den Daten auf Beschäftigtenebene steht das BHP auf Betriebsebene gegenüber. Das BHP umfasst alle Betriebe des gesamtdeutschen Raumes, die zum 30. Juni eines Jahres mindestens einen sozialversicherungspflichtig oder seit 1999 auch geringfügig Beschäftigten aufweisen. Eine detaillierte Darstellung des BHP findet sich in Hethey-Maier und Seth (2010). Auf Betriebsebene liegen zusätzlich die Survey-Daten des IAB-Betriebspanels vor. Einen Überblick über die Entstehungsgenese von der Stichprobenziehung bis zur Hochrechnung des IAB-Betriebspanels bieten u.a. Fischer et al. (2008).

Die insgesamt 289 im Rahmen des IAB-Betriebspanels im Jahr 2009 befragten Baubetriebe die unter die Mindestlohnregelung fallen, dienten als Grundlage für die Stichprobenziehung der eigenen Beschäftigtenbefragung im Bauhauptgewerbe. Die auf vollzeitbeschäftigte Arbeiter beschränkte Stichprobe wurde aus den IEB nach BetriebsgröBe disproportional geschichtet gezogen, um die Überrepräsentation von Großbetrieben im IAB-Betriebspanel auszugleichen. Insgesamt wurden 1521 telefonische Interviews realisiert. Themen der Befragung waren neben Angaben zur Erwerbshistorie seit 2009 (z.B. Art und Umfang der Beschäftigung, Angaben zu Betrieb und Baustelle), zur Soziodemographie und zum Einkommen auch die allgemeine Arbeitszufriedenheit, die Gewerkschaftsmitgliedschaft und die Mindestlohnregelungen.

Ergänzend zu den IAB-Daten wurden Daten aus dem Meldeverfahren zur Urlaubskasse der SOKA-Bau verwendet. Für das Projekt standen für Entsendearbeitnehmer die Daten für einen Zeitraum von 1997 bis 2011 und für inländische Beschäftigte für Juli 2009 bis Mai 2011 zur Verfügung. Auf Monatsbasis enthalten sie Bruttolohn, Beschäftigungstage und seit 2007 lohnzahlungspflichtige Stunden.

\section{Methodisches Vorgehen}

Die Untersuchung der Mindestlohneinführung im deutschen Bauhauptgewerbe erfolgt auf individueller, sektoraler sowie regionaler Ebene und unter der Prämisse, dass die Intervention als Quasi-Experiment angesehen werden kann. Es kommt v.a. ein Differenz-von-Differenzen-Ansatz zum Einsatz, der auf einer Einteilung in Treatment- und Kontrollgruppe beruht. Dies bedeutet, dass ein Teil der Beschäftigten als vom Mindestlohn betroffen angesehen werden kann (Treatmentgruppe), während eine passend definierte Kontrollgruppe als nicht betroffen gilt und somit zur Identifikation eines Mindestlohneffekts eingesetzt wird. Hierzu 
muss angenommen werden, dass sich die Treatmentgruppe in Abwesenheit einer Mindestlohnintervention analog zur Kontrollgruppe entwickelt hätte. Diese Annahme kann nicht getestet werden, wird allerdings glaubwürdiger, wenn beide Gruppen vor der Intervention möglichst ähnliche Eigenschaften aufweisen und sich vor der Mindestlohneinführung gleich entwickelt haben. Eine zweite Annahme ist die Nichtbetroffenheit der Kontrollgruppe von der Mindestlohnintervention. Innerhalb des Bauhauptgewerbes werden alle Beschäftigte der Treatmentgruppe zugeschlagen, die im Jahr vor der Mindestlohneinführung einen Stundenlohn unterhalb des neuen Mindestlohns erhalten. Die Kontrollgruppe wird durch die Beschäftigten gebildet, deren Stundenlohn zu diesem Zeitpunkt leicht über dem neuen Grenzwert liegt (siehe König und Möller 2009). In einem zweiten, alternativen Vorgehen beinhaltet die Treatmentgruppe alle Beschäftigte bzw. Betriebe im Bauhauptgewerbe und die Kontrollgruppe alle Beschäftigte bzw. Betriebe innerhalb einer Auswahl von Branchen, deren Lohn- und Beschäftigungsentwicklung in den Jahren vor der Mindestlohneinführung eine möglichst große Ähnlichkeit zum Bauhauptgewerbe aufwies. ${ }^{10}$

Die Schätzgleichung zur Untersuchung des Mindestlohneffekts auf die jeweils relevante Zielvariable entspricht dem Differenz-von-Differenzen (DvD) Ansatz (siehe Angrist und Krueger 1999; Lechner 2010) und hat für Beobachtung $i$ zum Zeitpunkt $t$ folgende Form:

$$
\begin{aligned}
Y_{i t}= & \alpha+\gamma D T_{i t}+\delta \text { post }_{t}+\theta(D T \times p o s t)_{i t} \\
& +\boldsymbol{X}_{i t} \boldsymbol{\beta}+\varepsilon_{i t} .
\end{aligned}
$$

Hierbei ist $D T$ eine Dummyvariable, die für Beobachtungen in der Treatmentgruppe den Wert 1 annimmt, ansonsten 0. Die Dummyvariable post markiert den Zeitraum des Post-Treatments, d.h. nach der Mindestlohneinführung bzw. -erhöhung 1, sonst 0 . Zudem enthält die Matrix $\boldsymbol{X}$ weitere Kontrollvariablen. $Y_{i t}$ ist die Ergebnisvariable für die jeweilige Untersuchung (z.B. Lohnwachstum, Beschäftigungssicherheit oder Beschäftigungsniveau der Person oder des Betriebs $i$ ). Der Koeffizient $\theta$ gibt dann den Effekt des Mindestlohns auf die Ergebnisvariable an. ${ }^{11}$

\footnotetext{
${ }^{10}$ Die Verflechtungen der Kontrollbranchen mit dem Bauhauptgewerbe werden berücksichtigt, indem sowohlfür Ost- als auch für Westdeutschland je eine dem Bauproduktionszyklus möglichst nahe (Malerund Lackierergewerbe), vorgelagerte (Herstellung von Kalksandstein), nachgelagerte (West: Herstellung von Holzmöbeln, Ost: Möbeltischlerei) sowie eine dem Produktionszyklus des Baugewerbes ferne Branche (West: Papier- und Pappeverarbeitung, Ost: Güterbeförderung mit Kraftfahrzeugen) verwendet wird. Eine detaillierte Darstellung des Auswahlverfahrens findet sich in IAB/RWI/ISG (2011).

${ }^{11}$ Der Endbericht enthält zudem Analysen der Mindestlohnerhöhungen unter Verwendung des von Dolton et al. (2012) vorgeschlagenen inkrementellen DvD (siehe IAB/RWI/ISG 2011). Diese werden hier aus Platzgründen nicht besprochen.
}

Da es sich bei der Einführung des Mindestlohns sozusagen um ein natürliches Experiment handelt und nicht alle Beschäftigte unter den Mindestlohn fallen, lässt sich die kausale Wirkung des Mindestlohns mit Hilfe von DvDAnsätzen analysieren. Entscheidend ist hierbei jedoch immer, dass die Annahme erfüllt ist, dass sich Treatment- und Kontrollgruppe gleich entwickelt hätten (,gäbe es keinen Mindestlohn") und dass die jeweilige Kontrollgruppe auch nicht indirekt beeinflusst wird. Diese Annahme lässt sich nicht testen und kann nur argumentativ anhand von Plausibilitätsüberlegungen geprüft werden, die in diesem Fall zufriedenstellend ausfallen. Grundsätzlich eignet sich der DvDAnsatz vor allem für kurzfristige Analysen, da die grundlegende Annahme der gleichen Entwicklung von Treatmentund Kontrollgruppe innerhalb eines kurzen Zeitraums eher erfüllt ist als in einer längerfristigen Betrachtung.

Um spezifische Hintergrundinformationen aus unterschiedlichen Perspektiven über die Besonderheiten der Baubranche, v.a. in Bezug auf die Mindestlohnregelungen und Bewertungen der Akteure zu erhalten, wurden neben den quantitativen Analysen Expertengespräche auf Grundlage des empirischen Konzepts des ,wissenschaftlichen Quellentextes“" geführt (Apel 2009). Die Gesprächspartner wurden aus den drei Akteursgruppen Arbeitgeberverbände, Gewerkschaften und Finanzkontrolle Schwarzarbeit ausgewählt. Bei den Tarifparteien wurden dabei sowohl Vertreter der jeweiligen Zentrale, als auch jeweils ein Regionalverband in Ost- und Westdeutschland befragt, um sowohl den unterschiedlichen Rahmenbedingungen in beiden Landesteilen als auch der strukturellen Organisation der Verbände gerecht zu werden. Bei der Auswahl der Regionalverbände wurden sowohl die Beschäftigtenzahl als auch der Branchenanteil an allen Beschäftigten herangezogen, um verhältnismäßig „baustarke“ Regionen auszuwählen.

Die Themenbereiche der Interviews umfassten die Bereiche Einführung und Auswirkungen des Mindestlohns, Tarifverhandlungen und Tarifparteien, Entsendungen, Umgehungsstrategien und Kontrollen. ${ }^{12}$

\section{Durchsetzbarkeit des Mindestlohns und Auswirkungen auf die Löhne}

Um mögliche Auswirkungen der Mindestlohnregelung untersuchen zu können, muss zuerst festgestellt werden, ob der Mindestlohn im Bauhauptgewerbe eingehalten wird und bindend ist bzw. wie viele Beschäftigte hiervon betroffen sind. Folglich werden in einem ersten Schritt mögliche Umgehungsstrategien untersucht, bevor Analysen der Lohnentwicklung erfolgen.

\footnotetext{
${ }^{12}$ Die Autorinnen und die Autoren danken den IAB-Projektmitarbeiterinnen und -mitarbeitern in den Stützpunkt-Agenturen für die außerordentliche Unterstützung bei der Vorbereitung und Durchführung der Expertengespräche.
} 
Umgehungsstrategien In den vergangen Jahren stieg die Zahl der aufgedeckten Verstöße gegen den Mindestlohn, was auf eine zunehmende Dichte und Effizienz der Kontrollen sowie den zusätzlichen Personalaufwand bei der Finanzkontrolle Schwarzarbeit zurückgeführt werden kann. In den Expertengesprächen wird dieser allgemeine Trend bestätigt, wobei die Durchsetzbarkeit des Mindestlohns im Bauhauptgewerbe noch weiter verbessert werden müsse. Eine klare Einschätzung bezüglich der Durchsetzbarkeit war allerdings keinem der Gesprächspartner möglich. ${ }^{13}$ Allerdings werden diverse illegale Ausweich- und Umgehungsstrategien als gebräuchlich beschrieben: Zum einen wären deutlich erhöhte Arbeitszeiten ohne Lohnausgleich die Praxis. Auch würde häufig nur ein Teil der Arbeit offiziell entlohnt und die andere schwarz ausbezahlt. Bei Kontrollen der Arbeitszeiten muss eine Diskrepanz zur tatsächlich erbrachten Leistung von der FKS nachgewiesen werden, was sehr schwierig sei. Zum anderen vergäben tarifgebundene Betriebe Aufträge für Bauleistungen an Subunternehmen aus Gewerken des Baunebengewerbes bzw. diese Subunternehmen täuschten anderweitige Arbeitsleistungen vor, um einen überwiegenden Teil ihrer wirtschaftlichen Aktivität dem Baunebengewerbe zuordnen zu können und somit offiziell nicht unter die Mindestlohnregelung zu fallen. Außerdem würden überhöhte Abzüge für Verwaltung, Transport, Unterkunft oder Verpflegung beobachtet sowie fiktive Abzüge für Schlechtarbeit oder für beschädigtes/zerstörtes Arbeitsmaterial. Darüber hinaus seien auch „Scheinselbständige“ zu beobachten, welche im Gegensatz zu Arbeitnehmern nicht unter dem Schutz des AEntG stehen, womit die Arbeitsbedingungen frei verhandelbar werden. Abschließend wurde noch die Beschäftigung ,klassischer“" Schwarzarbeiter in nicht unerheblichem Ausmaß erwähnt.

Aufgrund der mangelnden Datenlage beschränkt sich die Untersuchung möglicher Umgehungsstrategien im Rahmen dieses Projektes auf einen Aspekt: Der ALEED-BAU lässt die Untersuchung von (tatsächlichen oder vorgetäuschten) Verlagerungen des Tätigkeitsschwerpunkts bzw. einer Neuanmeldung des Betriebs zu mit dem Ziel, aus dem Geltungsbereich des TV Mindestlohn herauszufallen. ${ }^{14}$ Es zeigt sich jedoch im Vergleich zu den Kontrollbranchen kein nennenswerter Einfluss der Mindestlohneinführung auf Wechselaktivität des Wirtschaftszweiges oder auf Betriebsabmeldungen.

\footnotetext{
${ }^{13}$ Deskriptive Analysen der Stundenlohnverteilungen mit Hilfe der von SOKA-BAU bereit gestellten Daten zeigen, dass beinahe alle Personen mindestens den Mindestlohn I erhalten. Darüber hinaus scheinen auch Veränderungen der Lohnuntergrenzen zeitnah umgesetzt zu werden. Natürlich basieren diese Beobachtungen auf der Annahme, dass sowohl Lohnniveau als auch Stundenangaben korrekt übermittelt werden.

${ }^{14}$ Um tatsächliche Fluktuation in der Wirtschaftsklassifikation von rein statistischen Effekten abgrenzen zu können, kommt die Abgrenzungsmethode von Hethey und Schmieder (2010) zum Einsatz.
}

Deskription Der durchschnittliche nominale Brutto-Stundenlohn im westdeutschen Bauhauptgewerbe liegt über den gesamten Beobachtungszeitraum hinweg durchgehend oberhalb des Niveaus in Ostdeutschland (vgl. Abb. 1, linkes Panel). Der konjunkturelle Abschwung im deutschen Bauhauptgewerbe ab dem Jahr 1995 spiegelt sich in einer vorübergehenden Stagnation des Lohnwachstums wider. In Westdeutschland ist auch nach 2003 eine längere Phase der Stagnation zu beobachten. Das rechte Panel von Abb. 1 trägt die zeitliche Entwicklung der relativen Bedeutung des Mindestlohns ab, gemessen als Verhältnis zwischen Mindestlohn und Medianlohn (Kaitz-Index). Obwohl der Mindestlohn in den neuen Bundesländern absolut geringer ist als in den alten, ist die Betroffenheit aufgrund der Lohnverteilung deutlich höher. Zum Zeitpunkt der Mindestlohneinführung beträgt der Kaitz-Index fast 85 Prozent des Medianlohns in Ost- und 64 Prozent in Westdeutschland. In der weiteren Entwicklung sank die Betroffenheit aufgrund der Absenkung des Mindestlohns vorerst, um dann bis 1999 auf ca. 90 (Ost) bzw. 70 Prozent (West) anzusteigen. Anschließend verharrte der Kaitz-Index in Ostdeutschland bis 2003 auf diesem hohen Niveau, um dann auf ca. 83 Prozent abzusinken. In den alten Bundesländern hingegen stieg er bis 2009 auf etwa 74 Prozent an. Die Betroffenheit vom Mindestlohn II lag bei dessen Einführung 2003 bei 96 Prozent des Medianlohns (Ost) und bei 86 Prozent (West). Zum Zeitpunkt seiner Abschaffung in Ostdeutschland hatten sich diese Niveaus allerdings beinahe angeglichen. Im Verhältnis zu den Indexwerten für den Mindestlohn I zeigt sich grundsätzlich ein geringerer Abstand zwischen Ost und West. Insgesamt wird aber ein Angleichen der Mindestlohnbetroffenheit zwischen beiden Landesteilen deutlich.

Um ein differenzierteres Bild der Wirkungsweise des Mindestlohns auf die Lohnentwicklung zu erhalten, werden die Verteilungen der nominalen Stundenlöhne beider Landesteile mittels Kerndichteschätzung ermittelt und in einem Vorher-Nachher-Vergleich gegenüber gestellt. Abb. 2 stellt die Lohnverteilung vor der Einführung des Mindestlohns 1996 und neun Jahre später dar. ${ }^{15}$ Die Stundenlohnverteilung in den alten Bundesländern verändert sich über den gewählten Zeitraum in ihrer Form kaum. Eine naheliegende Erklärung besteht darin, dass 1996, dem Jahr vor der Mindestlohneinführung, gerade einmal vier Prozent der Löhne westdeutscher Bauarbeiter unterhalb des Mindestlohnniveaus lagen. Dies dürfte der überwiegend tariflichen Entlohnung in den alten Bundesländern geschuldet sein. Mit knapp 24 Prozent der betrachteten Personen ist die Betroffenheit von der Mindestlohneinführung in den neuen Bundesländern deutlich höher. Aus Abb. 2 wird ersichtlich, dass sich

\footnotetext{
${ }^{15}$ Aufgrund von Zensierung an der Beitragsbemessungsgrenze werden die Verteilungen zwar inklusive dieser Werte geschätzt, bei der grafischen Darstellung jedoch am 95ten Perzentil abgeschnitten.
} 


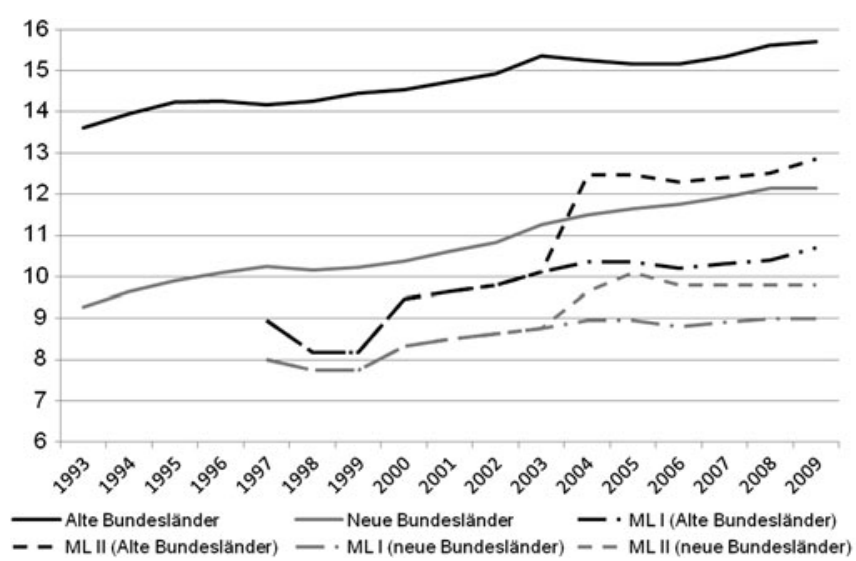

Abb. 1 (Links) Durchschnittliche Stundenlöhne im Bauhauptgewerbe in Euro. (Rechts) Relative Höhe der Mindestlöhne im Bauhauptgewerbe, Verhältnis des Mindestlohns zum mittleren Lohn (Median), in Prozent. Anmerkung: Die Einführung des Mindestlohns II wird im

die Verteilung der Stundenlöhne in den neuen Bundesländern innerhalb der neun Jahre sowohl von links als auch von rechts an die Mindestlohngrenzen angenähert hat. Insbesondere wird eine Verdichtung der Beobachtungen am Mindestlohn II offensichtlich, was für dessen hohe Bedeutung spricht. ${ }^{16}$ Von den Beschäftigten wird diese Reduktion der Lohnspreizung nach Aussage der Experten durchaus als negativ wahrgenommen.

Auswirkung des Mindestlohns auf das individuelle Lohnwachstum Um mögliche kausale Effekte der Mindestlohneinführung auf die Lohnverteilung der betrachteten Individuen identifizieren zu können, werden Differenz-vonDifferenzen Schätzungen durchgeführt (siehe Abschn. 4). Hierbei ist zu erwarten, dass von der Einführung einer Lohnuntergrenze betroffene Personen ceteris paribus im Vergleich zu nicht Betroffenen ein im Schnitt höheres Lohnwachstum erfahren.

Die Koeffizienten des Mindestlohneffekts auf das Lohnwachstum sind in Tab. 2 für die Mindestlohneinführung 1997 wiedergegeben. Die DvD-Effekte 1995 und 1996 stellen Placebo-Experimente dar, welche einen hypothetischen Mindestlohneffekt untersuchen, wenn dieser bereits ein bzw. zwei Jahre zuvor eingeführt worden wäre. Werden nicht betroffene Beschäftigte innerhalb des Bauhauptgewerbes als Kontrollgruppe gewählt (oberes Panel), zeigt sich abgesehen von 1995 in Ostdeutschland keine signifikante Abweichung, was die Annahme des Gleichlaufs in Abwesenheit der Mindestlohnintervention insgesamt weitgehend unterstützt.

\footnotetext{
${ }^{16}$ Ein Vergleich mit der Dynamik der Lohnverteilungen in den Kontrollbranchen deutet darauf hin, dass dieser Effekt nur im Bauhauptgewerbe auftritt. Dies wiederum spricht gegen Spillover Wirkungen des Mindestlohns im Bauhauptgewerbe auf andere Branchen.
}

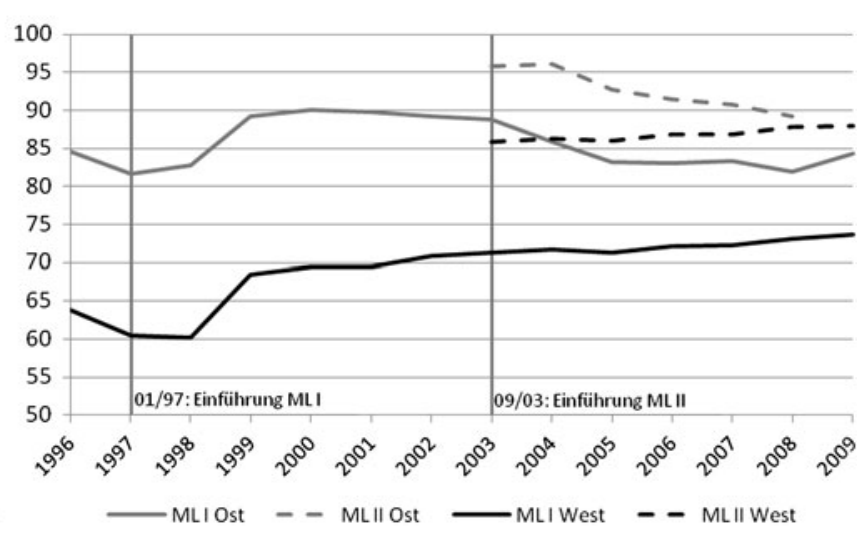

linken Panel erst 2004 sichtbar, da es sich hier um eine Stichtagsbetrachtung zum 30. Juni handelt. Der Mindestlohn II wurde allerdings erst zum 1. September 2003 eingeführt. Quelle: IAB/RWI/ISG (2011)

Durch die Einführung des Mindestlohns hat sich das Lohnwachstum von unmittelbar betroffenen Arbeitern im Bauhauptgewerbe signifikant um durchschnittlich 2,4 Prozentpunkte in Ost- und 1,4 Prozentpunkte in Westdeutschland erhöht. ${ }^{17}$ Die Placebo-Experimente mit einer Kontrollgruppe aus anderen Branchen scheitern jedoch in Ostdeutschland vollständig und sind in Westdeutschland lediglich 1996 nicht signifikant von Null verschieden (siehe Tab. 2, unteres Panel). Trotz der Ähnlichkeit in Lohn- und Beschäftigungsentwicklung vor der Mindestlohneinführung scheinen diese Branchen somit in diesem Fall keine geeignete Kontrollgruppe darzustellen.

Insgesamt gibt es Evidenz, dass die Mindestlohneinführung zu Lohnsteigerungen führte. Diese stellen sich in Ostdeutschland vergleichsweise deutlich dar, was auf die höhere Mindestlohnbetroffenheit zurückzuführen sein dürfte. Somit werden die bestehenden Ergebnisse der empirischen Mindestlohnliteratur für Deutschland bestätigt (vgl. König und Möller 2009; Rattenhuber 2011; Frings 2012).

Betriebliche Lohneffekte Auf Betriebsebene werden die Auswirkungen der Mindestlohnregelung auf die durchschnittliche Wachstumsrate des mittleren Bruttostundenlohns mittels einer Differenz-von-Differenzen Schätzungen untersucht. Wenn Betriebe einen Teil ihrer Beschäftigten vor der Einführung des Mindestlohns unterhalb der neuen Lohnuntergrenze entlohnen (Betroffenheit), wird für diese im Folgenden ein überdurchschnittlich hohes Lohnwachstum durch die Mindestlohneinführung erwartet. Die Kon-

\footnotetext{
${ }^{17}$ Diverse Spezifikationen bestätigen die Robustheit der dargestellten Ergebnisse gegenüber der Wahl der Mindestlohnabgrenzung, der Wahl von Stichtag- oder Zeitraumbetrachtung, der Wahl der Kontrollgruppen, gegenüber möglichen Spillover Effekten auf höher entlohnte Bauarbeiter sowie Messfehlern in den Stundenlöhnen.
} 

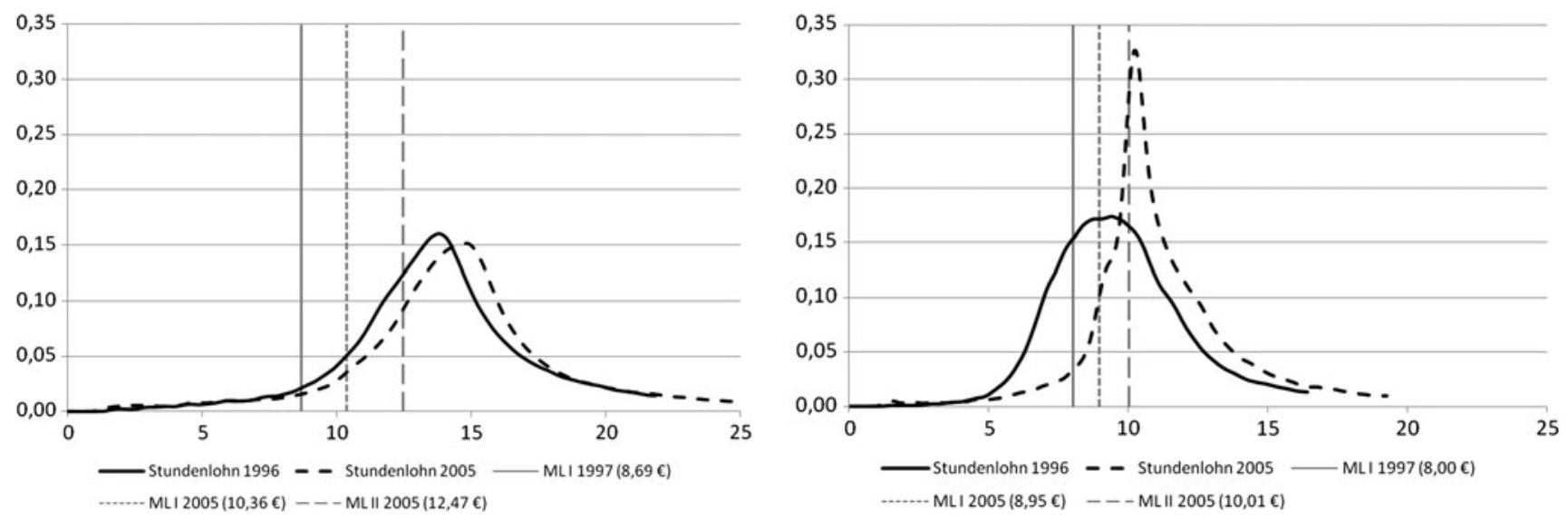

Abb. 2 (Links) Verteilung der Stundenlöhne 1996 und 2005 in Euro. Anmerkung: Der 1997 eingeführte Mindestlohn I galt bis August 2003 für alle Baubeschäftigte, ab September 2003 gilt für Fachkräfte der höhere Mindestlohn II. Quelle: IAB/RWI/ISG (2011)

Tab. 2 Lohnwachstum auf Personenebene für das Bauhauptgewerbe

\begin{tabular}{|c|c|c|c|c|c|c|c|c|}
\hline & \multicolumn{4}{|c|}{ Ostdeutschland } & \multicolumn{4}{|c|}{ Westdeutschland } \\
\hline & Koeffizient & & $R^{2}$ & $N$ & Koeffizient & & $R^{2}$ & $N$ \\
\hline \multicolumn{9}{|c|}{ Kontrollgruppe: Nicht-betroffene Personen innerhalb Bauhauptgewerbe } \\
\hline 1995 & $\begin{array}{c}0,005 \\
(2,32)\end{array}$ & $* *$ & 0,055 & 598476 & $\begin{array}{c}0,002 \\
(0,54)\end{array}$ & & 0,051 & 1234445 \\
\hline 1996 & $\begin{array}{l}-0,001 \\
(-0,50)\end{array}$ & & 0,049 & 615691 & $\begin{array}{c}-0,001 \\
(-0,20)\end{array}$ & & 0,053 & 1190107 \\
\hline 1997 (Einführung) & $\begin{array}{c}0,024 \\
(8,63)\end{array}$ & $* * *$ & 0,062 & 579057 & $\begin{array}{c}0,014 \\
(2,28)\end{array}$ & $* *$ & 0,049 & 1104828 \\
\hline \multicolumn{9}{|c|}{ Kontrollgruppe: Personen innerhalb aller Kontrollbranchen } \\
\hline 1995 & $\begin{array}{l}-0,007 \\
(-3,18)\end{array}$ & $* * *$ & 0,013 & 547590 & $\begin{array}{r}-0,021 \\
(-14,29)\end{array}$ & $* * *$ & 0,007 & 1306880 \\
\hline 1996 & $\begin{array}{c}0,016 \\
(6,86)\end{array}$ & $* * *$ & 0,007 & 564156 & $\begin{array}{c}0,002 \\
(1,36)\end{array}$ & & 0,008 & 1236680 \\
\hline 1997 (Einführung) & $\begin{array}{r}0,010 \\
(4,88)\end{array}$ & $* * *$ & 0,012 & 503375 & $\begin{array}{c}0,003 \\
(1,70)\end{array}$ & $*$ & 0,022 & 1097973 \\
\hline
\end{tabular}

Anmerkung: DvD-Schätzung. Signifikanzniveau: *** $1 \%, * * 5 \%, * 10 \% . t$-Werte in Klammern. Standardfehler auf Kreisebene geclustert. Es sind nur die DvD-Koeffizienten ausgewiesen. Weitere Kovariate sind: Treatmentgruppe, Treatmentjahr, Nationalität, Alter, Dauer der Betriebszugehörigkeit, Bildung, Beruf, Dauer der Wintererwerbstätigkeit, Anzahl der Arbeiter im Betrieb, Kreistyp und regionale Arbeitslosigkeit

Quelle: IAB/RWI/ISG (2011)

trollgruppe umfasst alle Betriebe, in denen alle Beschäftigten bereits über der Lohnuntergrenze entlohnt werden. Alle übrigen Betriebe des Bauhauptgewerbes sind in der Treatmentgruppe. Vor der Mindestlohneinführung 1997 sind in Westdeutschland 27 Prozent und in Ostdeutschland 71 Prozent der Betriebe betroffen. Die Effekte der Mindestlohneinführung in Ostdeutschland zeigen eine durchschnittliche Erhöhung des mittleren Lohnwachstums der betroffenen Betriebe um 0,7 Prozentpunkte zwischen 1996 und 1997 (Tab. 3). Dieser Effekt ist in den alten Bundesländern mit einem Prozentpunkt sogar etwas höher. Die Placebo-Tests gelingen.

\section{Wirkung auf Beschäftigung}

Im vorangegangenen Abschnitt wurde Evidenz für Auswirkungen des Mindestlohns auf die Lohnentwicklung aufgezeigt. Dieser könnte auch Beschäftigungseffekte zur Folge haben. Aus diesem Grund werden in diesem Abschnitt Beschäftigungsniveau, Einstellungen und Entlassungen sowie 
Tab. 3 Lohnwachstum auf Betriebsebene im Bauhauptgewerbe

\begin{tabular}{|c|c|c|c|c|c|c|c|c|}
\hline & \multicolumn{4}{|c|}{ Ostdeutschland } & \multicolumn{4}{|c|}{ Westdeutschland } \\
\hline & Koeffizient & & $R^{2}$ & $N$ & Koeffizient & & $R^{2}$ & $N$ \\
\hline \multicolumn{9}{|c|}{ Kontrollgruppe: Betriebe des Bauhauptgewerbes ohne betroffene Mitarbeiter } \\
\hline 1995 & $\begin{array}{r}0,001 \\
(0,17)\end{array}$ & & 0,023 & 30767 & $\begin{array}{l}-0,003 \\
(-1,79)\end{array}$ & * & 0,028 & 108698 \\
\hline 1996 & $\begin{array}{l}-0,001 \\
(-0,34)\end{array}$ & & 0,019 & 33688 & $\begin{array}{r}0,003 \\
(1,24)\end{array}$ & & 0,025 & 109512 \\
\hline 1997 (Einführung) & $\begin{array}{l}0,007 \\
(3,13)\end{array}$ & $* * *$ & 0,025 & 34848 & $\begin{array}{c}0,010 \\
(4,54)\end{array}$ & $* * *$ & 0,027 & 108096 \\
\hline
\end{tabular}

Anmerkung: DvD-Schätzung. Signifikanzniveau: *** $1 \%, * * 5 \%, * 10 \% . t$-Werte in Klammern. Standardfehler auf Ebene der Arbeitsmarktregionen geclustert. Es sind nur die DvD-Koeffizienten ausgewiesen. Weitere Kovariate sind: Treatmentgruppe,Treatmentjahr, Betriebsgröße (Anzahl der Arbeiter, Anzahl der Angestellten, Anzahl der Auszubildenden), regionale Arbeitslosigkeit und Kreistyp

Quelle: IAB/RWI/ISG (2011)

Marktmacht und Beschäftigtenstruktur näher untersucht. Die Analyse der Entwicklung von Entsendungen im Bauhauptgewerbe kann dabei aufgrund fehlender Daten vor der Mindestlohneinführung nur deskriptiv erfolgen. Die Untersuchungen berücksichtigen daher keine möglichen Substitutionswirkungen zwischen entsandten und inländischen Arbeitskräften.

Mit rund sechs Prozent beschäftigte das Bauhauptgewerbe 1995 einen substantiellen Anteil aller Beschäftigten in Deutschland (vgl. Abb. 3). Dieser fiel bis 2008 jedoch kontinuierlich bis auf 2,3 Prozent ab. Waren zur Hochphase des Baubooms 1995 noch über einer Million Personen im westdeutschen und 460000 Personen im ostdeutschen Bauhauptgewerbe beschäftigt, so betrug deren Zahl 2006 nur noch 528000 in den alten bzw. 222000 in den neuen Bundesländern.

Die Einführung des Mindestlohns fiel somit in eine Zeit grundlegender Veränderungen innerhalb des Bauhauptgewerbes. Dies macht eine Abschätzung kausaler Wirkungsweisen mit bloßen deskriptiven Mitteln unmöglich.

Nachfolgend wird auf Ebene von Arbeitsmarktregionen (vgl. Eckey et al. 2007) durch Differenz-von-Differenzen Schätzungen untersucht, wie die Mindestlohneinführung die Beschäftigung im Bauhauptgewerbe im Vergleich zu den Kontrollbranchen beeinflusst hat.

Die Ergebnisse der DvD-Schätzungen zeigen insignifikante Koeffizienten bei den Placebo-Tests (vgl. Tab. 4). Demnach ist die notwendige Annahme gleichlaufender Trends vor dem eigentlichen Experiment erfüllt und eine kausale Interpretation möglich. Die DvD-Koeffizienten der Mindestlohneinführung zeigen keine signifikanten Effekte, weder für Ost- noch für Westdeutschland. Auf Ebene der Regionen können demnach keine Auswirkungen der Mindestlohneinführung auf das Beschäftigungsniveau festgestellt werden.
Neben Auswirkungen auf das Beschäftigungsniveau könnte es auch Auswirkungen des Mindestlohns auf Einstellungen und Entlassungen geben.

Die Untersuchung der Mindestlohneinführung erfolgt mittels Differenz-von-Differenzen Schätzung auf Basis eines linearen Wahrscheinlichkeitsmodells, wobei die Trennungswahrscheinlichkeit auf Personenebene und die Einstellungswahrscheinlichkeit auf betrieblicher Ebene gemessen wird. Zieht man Arbeiter im Bauhauptgewerbe, die vor Einführung des Mindestlohns oberhalb dieser Lohnuntergrenze entlohnt werden, als Kontrollgruppe heran, gelingt das Placebo-Experiment in Ost- und scheitert in Westdeutschland. Der als signifikant negativ gemessene Mindestlohneffekt auf die individuelle Trennungswahrscheinlichkeit in Westdeutschland ist demnach nicht kausal interpretierbar. In Ostdeutschland hingegen wird kein signifikant von Null verschiedener Effekt als Folge der Einführung ersichtlich (vgl. Tab. 5). ${ }^{18}$ Dies spricht gegen einen Effekt des Mindestlohns auf die individuelle Trennungswahrscheinlichkeit.

Ergänzend wurde ein Ansatz aufgestellt, welcher Arbeiter aus den Kontrollbranchen als Kontrollgruppe wählt. Für die alten Bundesländer gelingt hier das Placebo-Experiment nur für die vor- und nachgelagerte Kontrollbranche. In diesen Fällen ergibt sich kein signifikanter Effekt des eigentlichen Treatments. Für die neuen Bundesländer zeigt sich in der Regel ein analoges Bild: Gelingt das PlaceboExperiment, werden keine signifikanten Effekte gemessen. Einzige Ausnahme stellt die nachgelagerte Branche in Ostdeutschland dar, für die das Placebo-Experiment gelingt und sich ein signifikant negativer Effekt der Mindestlohneinführung auf die individuelle Trennungswahrscheinlichkeit von

\footnotetext{
${ }^{18}$ Die Ergebnisse erweisen sich als robust gegenüber Variation der Kontrollvariablen, Variation der Kontrollgruppe bezüglich Ausschluss höher Entlohnter und geringfügig über Mindestlohn entlohnter Bauarbeiter.
} 
Abb. 3 Entwicklung der Beschäftigung im Bauhauptgewerbe 1995 bis 2010, West- und Ostdeutschland, Personen in 1.000. Quelle:

Sachverständigenrat zur Begutachtung der gesamtwirtschaftlichen Entwicklung (2011); eigene Darstellung

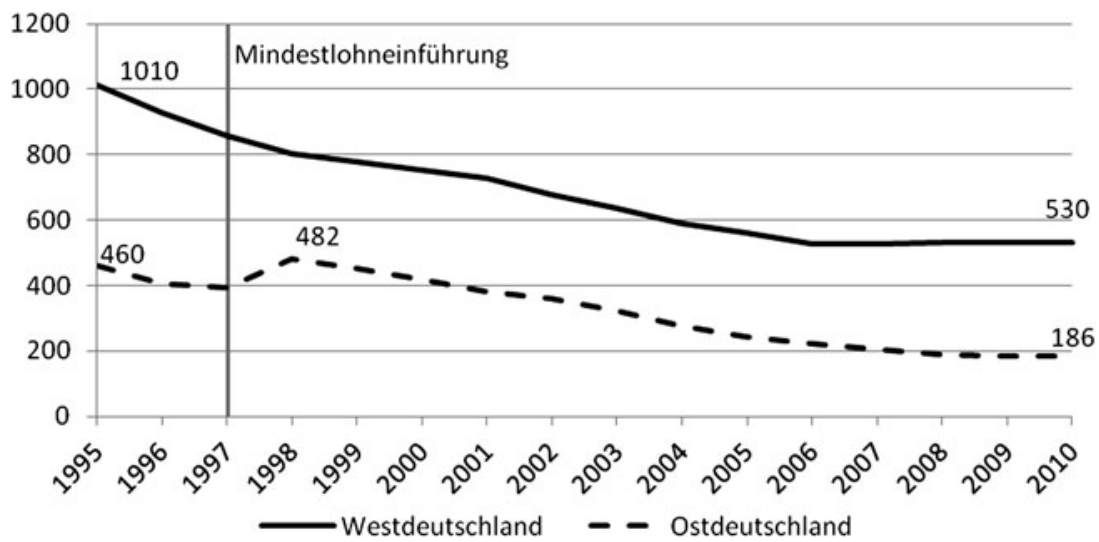

Tab. 4 Beschäftigungsniveau auf Regionsebene

\begin{tabular}{|c|c|c|c|c|c|c|}
\hline & \multicolumn{3}{|c|}{ Ostdeutschland } & \multicolumn{3}{|c|}{ Westdeutschland } \\
\hline & Koeffizient & $R^{2}$ & $N$ & Koeffizient & $R^{2}$ & $N$ \\
\hline \multicolumn{7}{|c|}{ Kontrollgruppe: Alle Kontrollbranchen } \\
\hline 1995 & $\begin{array}{l}-0,048 \\
(-0,53)\end{array}$ & 0,846 & 340 & $\begin{array}{l}-0,031 \\
(-0,30)\end{array}$ & 0,564 & 1079 \\
\hline 1996 & $\begin{array}{l}-0,079 \\
(-0,88)\end{array}$ & 0,844 & 340 & $\begin{array}{l}-0,038 \\
(-0,37)\end{array}$ & 0,565 & 1078 \\
\hline 1997 (Einführung) & $\begin{array}{l}-0,061 \\
(-0,67)\end{array}$ & 0,829 & 340 & $\begin{array}{l}-0,062 \\
(-0,61)\end{array}$ & 0,564 & 1075 \\
\hline
\end{tabular}

Anmerkung: DvD-Schätzung. Signifikanzniveau: *** $1 \%, * * 5 \%, * 10 \% . t$-Werte in Klammern. Weitere Kovariate sind: mittlerer Stundenlohn, Bevölkerung und regionale Arbeitslosigkeit

Quelle: IAB/RWI/ISG (2011)

sechs Prozentpunkten ergibt. Letztendlich kann geschlussfolgert werden, dass diese Untersuchung kaum kurzfristige Effekte der Mindestlohneinführung auf die durchschnittlichen Trennungen der zunächst unter Mindestlohn entlohnten Arbeiter im Bauhauptgewerbe erkennen lässt.

Die DvD-Koeffizienten der Mindestlohneinführung auf die Einstellungswahrscheinlichkeit auf Betriebsebene sind in Tab. 6 dargestellt.

Eine Einstellung ergibt sich, wenn eine Person entweder aus einem anderen Betrieb oder aus der Nichterwerbstätigkeit in das Bauhauptgewerbe wechselt. ${ }^{19}$ Die PlaceboTests für Ostdeutschland bestätigen einen parallelen Trend zwischen der Einstellungswahrscheinlichkeit im Bauhauptgewerbe und den Kontrollbranchen vor Mindestlohneinführung. Für Westdeutschland wird diese Hypothese abgelehnt. In den neuen Bundesländern hatte die Mindestlohneinführung für betroffene Betriebe einen signifikant leicht dämpfenden Effekt auf die Einstellungswahrscheinlichkeit.

\footnotetext{
${ }^{19}$ Unabhängig von der Beobachtungsebene sind alle Ergebnisse robust gegenüber der Berücksichtigung von ,recalls“, definiert als Wiedereinstellung bei einem Arbeitgeber nach weniger als 91 Tagen Beschäftigungsunterbrechung.
}

\section{Marktmacht und Entsendungen}

Marktmacht Die Beschäftigungswirkung eines Mindestlohns hängt sehr stark von der Marktmacht in einer Branche ab. Um die Verhandlungsmacht der Arbeitnehmer gegenüber den Arbeitgebern zu erfassen, wird der Anteil der Wechsler aus der Nicht-Erwerbstätigkeit in einen Betrieb des Bauhauptgewerbes an den gesamten Einstellungen im Bauhauptgewerbe aus dem ALEED-Bau berechnet. Manning (2003) zufolge kann dieser Indikator als Grad des Wettbewerbs unter Arbeitgebern um Beschäftigte interpretiert werden: Steht den Firmen ein großer Pool von arbeitslosen Fachkräften zur Besetzung offener Stellen zur Verfügung, ist die Verhandlungsmacht der Arbeitnehmer relativ gering und die Marktmacht der Firmen auf dem Arbeitsmarkt relativ hoch. Im Gegensatz dazu deuten häufige Wechsel zwischen verschiedenen Arbeitgebern ohne zwischenzeitliche Arbeitslosigkeitsphasen auf einen starken Wettbewerb von Firmen um Beschäftigte hin und die Marktmacht der Arbeitgeber kann als relativ gering eingestuft werden. Die Berechnung erfolgt getrennt nach neuen und alten Bundesländern und exklusive „recalls“, also Wiedereinstellungen in dersel- 
Tab. 5 Trennungswahrscheinlichkeit auf Personenebene

\begin{tabular}{|c|c|c|c|c|c|c|c|c|}
\hline & \multicolumn{4}{|c|}{ Ostdeutschland } & \multicolumn{4}{|c|}{ Westdeutschland } \\
\hline & Koeffizient & & $R^{2}$ & $N$ & Koeffizient & & $R^{2}$ & $N$ \\
\hline \multicolumn{9}{|c|}{ Kontrollgruppe: Nicht-betroffene Personen innerhalb Bauhauptgewerbe } \\
\hline 1996 & $\begin{array}{l}-0,014 \\
(-1,06)\end{array}$ & & 0,090 & 757021 & $\begin{array}{c}-0,067 \\
(-9,47)\end{array}$ & $* * *$ & 0,117 & 1394535 \\
\hline 1997 (Einführung) & $\begin{array}{c}0,001 \\
(0,11)\end{array}$ & & 0,108 & 737489 & $\begin{array}{l}-0,019 \\
(-2,65)\end{array}$ & $* * *$ & 0,111 & 1319818 \\
\hline \multicolumn{9}{|c|}{ Kontrollgruppe: Vorgelagerte Branche } \\
\hline 1996 & $\begin{array}{c}0,028 \\
(1,48)\end{array}$ & & 0,081 & 794395 & $\begin{array}{l}-0,004 \\
(-0,45)\end{array}$ & & 0,105 & 1501673 \\
\hline 1997 (Einführung) & $\begin{array}{l}-0,008 \\
(-0,50)\end{array}$ & & 0,093 & 775392 & $\begin{array}{l}-0,001 \\
(-0,12)\end{array}$ & & 0,103 & 1422930 \\
\hline \multicolumn{9}{|c|}{ Kontrollgruppe: Nachgelagerte Branche } \\
\hline 1996 & $\begin{array}{c}0,020 \\
(1,37)\end{array}$ & & 0,090 & 778821 & $\begin{array}{l}-0,001 \\
(-0,15)\end{array}$ & & 0,106 & 1531116 \\
\hline 1997 (Einführung) & $\begin{array}{l}-0,060 \\
(-3,24)\end{array}$ & $* * *$ & 0,091 & 759673 & $\begin{array}{c}0,005 \\
(0,56)\end{array}$ & & 0,104 & 1449150 \\
\hline \multicolumn{9}{|c|}{ Kontrollgruppe: Baunahe Branche } \\
\hline 1996 & $\begin{array}{r}0,015 \\
(1,56)\end{array}$ & & 0,082 & 819137 & $\begin{array}{c}0,014 \\
(4,02)\end{array}$ & $* * *$ & 0,108 & 1593291 \\
\hline 1997 (Einführung) & $\begin{array}{c}0,001 \\
(0,11)\end{array}$ & & 0,094 & 802260 & $\begin{array}{l}-0,001 \\
(-0,29)\end{array}$ & & 0,106 & 1514907 \\
\hline \multicolumn{9}{|c|}{ Kontrollgruppe: Bauferne Branche } \\
\hline 1996 & $\begin{array}{c}0,086 \\
(8,79)\end{array}$ & $* * *$ & 0,078 & 825022 & $\begin{array}{r}0,047 \\
(3,46)\end{array}$ & $* * *$ & 0,107 & 1427446 \\
\hline 1997 (Einführung) & $\begin{array}{l}-0,034 \\
(-2,13)\end{array}$ & $* *$ & 0,089 & 809998 & $\begin{array}{l}-0,079 \\
(-2,79)\end{array}$ & $* * *$ & 0,104 & 1351763 \\
\hline
\end{tabular}

Anmerkung: DvD-Schätzung. Signifikanzniveau: *** $1 \%, * * 5 \%, * 10 \% . t$-Werte in Klammern. Standardfehler auf Kreisebene geclustert. Es sind nur die DvD-Koeffizienten dargestellt. Weitere Kovariate sind: Treatmentgruppe, Treatmentjahr, Nationalität, Alter, Dauer der Betriebszugehörigkeit, Bildung, Dauer der Wintererwerbstätigkeit, Anzahl der Arbeiter im Betrieb, Kreistyp und regionale Arbeitslosigkeit

Quelle: IAB/RWI/ISG (2011)

Tab. 6 Einstellungswahrscheinlichkeit auf Betriebsebene

\begin{tabular}{|c|c|c|c|c|c|c|c|c|}
\hline & \multicolumn{4}{|c|}{ Ostdeutschland } & \multicolumn{4}{|c|}{ Westdeutschland } \\
\hline & Koeffizient & & $R^{2}$ & $N$ & Koeffizient & & $R^{2}$ & $N$ \\
\hline \multicolumn{9}{|c|}{ Kontrollgruppe: Alle Kontrollbranchen } \\
\hline 1995 & $\begin{array}{l}-0,002 \\
(-0,28)\end{array}$ & & 0,111 & 67894 & $\begin{array}{l}-0,010 \\
(-1,71)\end{array}$ & $*$ & 0,124 & 178290 \\
\hline 1996 & $\begin{array}{r}0,007 \\
(0,98)\end{array}$ & & 0,144 & 72334 & $\begin{array}{c}0,016 \\
(3,01)\end{array}$ & $* * *$ & 0,158 & 178681 \\
\hline 1997 (Einführung) & $\begin{array}{l}-0,027 \\
(-3,42)\end{array}$ & $* * *$ & 0,194 & 74903 & $\begin{array}{l}-0,014 \\
(-2,76)\end{array}$ & $* * *$ & 0,197 & 177142 \\
\hline
\end{tabular}

Anmerkung: DvD-Schätzung. Signifikanzniveau: *** $1 \%, * * 5 \%, 10 \% . t$-Werte in Klammern. Es sind nur die DvD-Koeffizienten dargestellt. Weitere Kovariate sind: Treatmentgruppe, Treatmentjahr, Betriebsgröße (Anzahl der Arbeiter, Anzahl der Angestellten, Anzahl der Auszubildenden), regionale Arbeitslosigkeit und Kreistyp

Quelle: IAB/RWI/ISG (2011) 
ben Firma nach einer Periode der Nichterwerbstätigkeit, die höchstens 90 Tage andauert. ${ }^{20}$

Zwischen 1993 und 2009 liegt der durchschnittliche Wert des Marktmachtindikators bei 83 Prozent in Ost- und 84 Prozent in Westdeutschland. Stellt man einen Wert von 47,5 Prozent für männliche Arbeitnehmer in Deutschland gegenüber (siehe Hirsch 2010: 172), zeigt sich das vergleichsweise hohe Maß an Marktmacht der Arbeitgeber im Bauhauptgewerbe. In der zeitlichen Entwicklung wird ersichtlich, dass sich die Marktmacht beider Landesteile seit den späten 1990er Jahren gleichläufig entwickelt und in den neuen Bundesländern etwas höher liegt. Zwischen 1993 und 1995 war die Marktmacht hingegen in Ostdeutschland deutlich geringer, was auch auf den Bauboom im Zuge der deutschen Wiedervereinigung zurückzuführen ist. Die rein deskriptive Betrachtung lässt allerdings keinen Einfluss der Mindestlohnregelung auf die Entwicklung des Marktmachtindikators erkennen, der tendenziell dem Wirtschaftszyklus folgt. Tiefergehende kausale Aussagen sind für diesen Indikator nicht möglich.

Entsendungen In den Expertengesprächen wird die Vielzahl der im Zuge des ostdeutschen Baubooms nach Deutschland entsandten Arbeitnehmer als maßgeblicher Grund für die Einführung des Mindestlohns gesehen. ${ }^{21}$ Die Mindestlohneinführung könnte demnach auch Beschäftigungseffekte auf diese Arbeitnehmergruppe ausüben, da deren Einsatz Worthmann und Zühlke-Robinet (2003: 98) zufolge als das „Pendant zur Standortverlagerung“ betrachtet werden kann.

Entsendungen aus der EU im Rahmen der uneingeschränkten Dienstleistungsfreiheit werden erst ab 1997 von der zuständigen Behörde der Zollverwaltung und durch SOKA-BAU erfasst. ${ }^{22}$ Bezüglich der Zahl an Werkvertragsarbeitnehmern aus MOE-Staaten sowie der Türkei liegen Daten der Bundesagentur für Arbeit seit 1990 vor. Die Zahl der MOE-Entsendungen zeigt einen deutlichen Anstieg von 1990 bis 1992. Noch während des anhaltenden Baubooms (und somit vor Einführung des Mindestlohns) reduziert sie sich jedoch erneut. Da das Bestehen bilateraler Werkvertragsabkommen für diese Gruppe prinzipiell deutsche Arbeitsbedingungen vorschreibt, wäre eine mögliche Hypothese, dass in der Hauptphase des Baubooms eine Substitution hin zu Entsendungen aus dem EWR erfolgt ist. Dies kann jedoch anhand des verfügbaren Datenmaterials nicht geprüft werden.

Seit 1997 sind sämtliche Entsendezahlen rückläufig (mit leichter Erholung ab 2006). Ob dies auf die konjunkturelle Entwicklung oder aber den Mindestlohn zurückzuführen

\footnotetext{
${ }^{20}$ Die Ergebnisse sind allerdings robust gegenüber der Berücksichtigung von recalls.

${ }^{21}$ Vgl. hierzu auch Kahmann (2006: 187).

${ }^{22}$ Für den Zeitraum vor 1997 liegen bezüglich Entsendezahlen aus dem EWR keine verlässlichen Daten vor.
}

ist, kann aufgrund einer fehlenden Kontrollgruppe sowie der Nicht-Verfügbarkeit von Daten über EU-Entsendungen vor 1997 nicht geklärt werden.

\section{Wirkung auf Arbeitnehmerschutz und Wettbewerb}

Die Auswirkungen einer Mindestlohneinführung müssen nicht auf Löhne und Beschäftigung beschränkt bleiben. Einerseits könnten Mehrkosten durch steigende Lohnkosten zulasten von Qualifizierung und anderen Arbeitsbedingungen gehen. Andererseits sind auch wettbewerbsverzerrende Auswirkungen denkbar. Aus diesem Grund werden hier die Wirkungen der Mindestlohneinführung auf die Aspekte betriebliche Weiterbildung, Befristungen, Überstunden, allgemeine Arbeitszufriedenheit und Gewerkschaftsmitgliedschaft dargestellt. Stellvertretend für die Wettbewerbswirkung von Mindestlöhnen werden Betriebsgrößenänderungen, Performanceentwicklung, Marktein- und Austrittsraten, Preisentwicklung und Tarifbindungsgrad untersucht.

Durch die Einführung eines Mindestlohns lassen sich zwei mögliche Auswirkungen auf Weiterbildungsmaßnahmen erwarten. Einerseits kann angenommen werden, dass die steigenden Lohnkosten durch geringere Aufwendungen für die Weiterqualifizierung von Mitarbeitern und damit einhergehenden Ersparnissen kompensiert werden könnten. Andererseits wird durch den Mindestlohn die Arbeitskraft teurer. Das könnte dazu führen, dass ihre Produktivität unter dem gezahlten Mindestlohn liegt. Mit gezielten Weiterbildungsmaßnahmen könnten Arbeitgeber möglicherweise die Produktivität ihrer Arbeitnehmer steigern und so dem Kostenanstieg entgegenwirken. Ob und welche Wirkungen eintreten, wurde auf Betriebsebene anhand des IAB-Betriebspanels untersucht. Die Beschäftigtenbefragung zeigt aber, dass Weiterqualifizierung im Bauhauptgewerbe einen geringen Stellenwert besitzt. Nur rund 35 Prozent der westdeutschen und 29 Prozent der ostdeutschen Arbeiter im Bau haben 2010 an einer Weiterbildung teilgenommen. In den Expertengesprächen werden ein geringes Interesse der Arbeitnehmer und die von Kleinbetrieben dominierte Betriebsstruktur als Gründe für die geringe Weiterbildungsrate angeführt. Mindestlohneinflüsse werden hier nicht gesehen. Vielmehr könnten der demographische Wandel und der drohende Fachkräftemangel die Bedeutung von Weiterbildung im Bau erhöhen. Die auf der Betriebsebene durchgeführten DvD-Ansätze mit verschiedenen Kontrollgruppen haben ebenfalls keine signifikanten Änderungen bei den Weiterbildungskennzahlen durch die Mindestlohneinführung gezeigt, ${ }^{23}$ was auf geringe Wirkungen des Mindestlohns hindeutet. Der Aussagegehalt wird durch die kleinen Fallzahlen auf Betriebsebene allerdings stark beeinträchtigt.

\footnotetext{
${ }^{23}$ Eine ausführliche Darstellung der Methoden und Ergebnisse findet sich in IAB/RWI/ISG (2011).
} 
Die Befristung von Arbeitsverträgen stellt eine weitere Möglichkeit dar, Lohnkostensteigerungen durch die Mindestlohneinführung zu kompensieren. Mit Regelungen wie dem Schlechtwettergeld (bis 1995) oder dem SaisonKurzarbeitergeld (seit Winter 2006/2007) existieren im Bauhauptgewerbe jedoch auch andere Instrumente zur Flexibilisierung von Beschäftigung. Entsprechend wenig Bedeutung kommt den befristeten Beschäftigungsverhältnissen zu. Mit fünf Prozent liegt der Anteil befristet Beschäftigter in der Beschäftigtenbefragung ${ }^{24}$ sogar unter dem bundesweiten Durchschnitt von 8,9 Prozent (Destatis 2011). Die Kausalanalyse auf Betriebsebene zeigt zudem, dass sich die Bedeutung befristeter Beschäftigungsverhältnisse durch die Mindestlohneinführung nicht verändert hat.

Weitaus größere Bedeutung als Befristungen kommt laut Experten dem Umgang mit Überstunden im Bauhauptgewerbe zu. Um Kosten zu sparen, würde Mehrarbeit zum Teil gar nicht oder nur schwarz entlohnt, eine These, die mit den vorhandenen Daten nicht überprüfbar ist. Es ist jedoch auch ein legaler Überstundenrückgang mit der Mindestlohneinführung vorstellbar, der auf Substitutionseffekten beruhen kann und in den Daten beobachtbar ist. Für die kausale Analyse wurden sowohl die Angabe der Betriebe, ob im Geschäftsjahr Überstunden geleistet wurden und die Gesamtüberstunden verwendet. Beide Größen zeigten keine signifikanten Änderungen durch die Mindestlohneinführung.

Im nächsten Schritt wird die subjektive Sicht der Arbeitnehmer in den Fokus der Untersuchung gerückt. Wenn sich der Mindestlohn auf die Zufriedenheit auswirkt, sind zwei Entwicklungen denkbar. Zum einen kann sich die Zufriedenheit insbesondere bei den vom Mindestlohn betroffenen Beschäftigten durch die damit verbundene Lohnsteigerung verbessern. Auf der anderen Seite hat die Mindestlohneinführung, wie gezeigt, in den ostdeutschen Bundesländern zu einer Verringerung der Lohnabstände geführt, was Quelle für Unzufriedenheit vor allem unter qualifizierten Beschäftigten sein kann. Die Analysen in diesem Bereich stammen ausschließlich aus der Beschäftigtenbefragung. Sie zeigen allgemein ein recht positives Bild der Zufriedenheit zum Befragungszeitpunkt. Etwa 70 Prozent aller Befragten geben an, mit der Art ihrer Tätigkeit, den Arbeitsschutzmaßnahmen und dem Betriebsklima zufrieden oder sogar sehr zufrieden zu sein. Eher schlechter bewertet werden hingegen zum einen das Einkommen und zum anderen Arbeitstempo und Termindruck, mit denen nur ca. 30 Prozent zufrieden sind. Viele der Befragten, insbesondere in Ostdeutschland und unter den Jüngeren, empfinden allerdings die aktuelle Tarifstruktur als ungerecht. Die Mehrheit der Befragten ist jedoch nicht der Meinung, dass die Gewerkschaft durch die Mindestlöhne an Bedeutung verloren hat. Aus den Analysen zu Weiterbildung, Befristungen, Überstunden,

\footnotetext{
${ }^{24}$ Angabe aus der Beschäftigtenbefragung.
}

Arbeitszufriedenheit und Gewerkschaftsmitgliedschaft lässt sich insgesamt nicht ableiten, dass der Mindestlohn die Arbeitsbedingungen im Bauhauptgewerbe entscheidend verändert hat.

Nach dem Ende des Aufschwungs in der Bauwirtschaft 1995 kam es zu einem Anstieg der Zahl der Betriebe im Bauhauptgewerbe von 74000 auf über 81 000, der hauptsächlich auf einen Zuwachs bei den Kleinst- und Kleinbetrieben und zu Lasten größerer Betriebe zurückzuführen ist. Als Gründe für diese Entwicklung kommen einerseits Betriebsneugründungen oder Ausgliederungen, andererseits aber auch die Entlassung von Mitarbeitern, möglicherweise bedingt durch den Mindestlohn, in Frage. Der Einfluss der Mindestlohneinführung auf diese Entwicklung wird mittels DvD-Schätzung anhand der ALEED-Bau-Daten untersucht. In den neuen Bundesländern scheint die Mindestlohneinführung 1997 einen leicht positiven Effekt auf die Entwicklung der Betriebsgröße gehabt zu haben.

Die Marktein- und Marktaustritte von Betrieben spielen nicht nur bei der Betriebsgrößenstruktur eine Rolle, sie können auch direkte Folge mindestlohnbedingter Lohnkostensteigerungen sein. Die gestiegenen Lohnkosten könnten einerseits zu einer erhöhten Insolvenz- und Marktaustrittsrate geführt und die Zahl der Neugründungen verringert haben. Andererseits ist zu erwarten, dass die Gründung von Kleinstbetrieben zugenommen hat, da Selbstständige nicht unter die Mindestlohnregelung fallen. Selbständige können in den Daten nicht beobachtet werden, was die Analyse auf die erste These beschränkt. Die Insolvenzwahrscheinlichkeit lag zwischen 1995 und 2005 in den neuen Bundesländern mit bis zu elf Prozent deutlich über der in den alten Bundesländern. Erst seit 2005 ist sie in etwa auf dem westdeutschen Niveau von drei bis vier Prozent. Die Ein- und Austrittsraten der Betriebe, definiert als Anteil der Betriebe, die im Vorjahr noch nicht bzw. im Jahr später nicht mehr existieren, entsprechen sich in Westdeutschland in den meisten Jahren in etwa. In Ostdeutschland sind sowohl Markteintritts- als auch Marktaustrittsrate höher als in Westdeutschland. In den vergangenen Jahren sind die Raten allerdings gesunken und nur noch leicht über dem Westniveau. Auch zwischen den beiden Raten sind in Ostdeutschland größere Unterschiede zu erkennen. Für die Marktein- und Marktaustrittswahrscheinlichkeit der Betriebe scheinen sowohl Betriebe in den Kontrollbranchen bereits vor der Mindestlohneinführung unterschiedlich in ihrer Entwicklung zu Betrieben des Bauhauptgewerbes. Auch Betriebe, die vom Mindestlohn betroffen sind unterscheiden sich bereits in den Jahren vor der Einführung von den nicht betroffenen Betrieben, worauf signifikante Placebo-Tests hindeuten. Daher werden Kausalanalysen der Marktein- und Austrittsraten nicht dargestellt.

Direkt nach der Wiedervereinigung führte der Bauboom zu einem starken aber kurzen Anstieg der Umsätze und Investitionen in der Baubranche bis 1995. Danach fielen die 
Werte teilweise unter das Niveau von 1991. Erst ab ca. 2005 konnte dieser Negativtrend, der in Ostdeutschland sehr viel stärker ausgeprägt war, gestoppt werden. Die Mindestlöhne haben nach Expertenmeinung in dieser allgemeinen Krisensituation kaum eine Wirkung gehabt. Die Kausalanalyse bekräftigt diese Aussage: weder auf Umsatz noch auf Investitionen lassen sich im IAB-Betriebspanel signifikante Effekte durch die Mindestlohneinführung feststellen.

Die Krise der Bauwirtschaft hat laut Expertengespräche zudem den Wettbewerbs- und Preisdruck im Bauhauptgewerbe erhöht. Die Lohnuntergrenze wird in diesem Zusammenhang positiv gesehen, da sie im personalkostenintensiven Bausektor einen Lohnwettbewerb zu Lasten der Arbeitnehmer verhindert. Eine Kausalanalyse der Mindestlohneffekte auf die Preisentwicklung im Bauhauptgewerbe ist aufgrund mangelnder Daten nicht möglich. Die deskriptiven Analysen deuten darauf hin, dass mögliche gestiegene Lohnkosten durch den Mindestlohn nicht auf die Preise überwälzt wurden. Bis 1999 entwickeln sich Baupreise und Inputfaktoren parallel. Danach kommt es zu einer Entkopplung, mit sehr viel stärker steigenden Kosten für Inputfaktoren relativ zu den Baupreisen. Erst 2005 mit der konjunkturellen Erholung steigen die Baupreise wieder stärker an, wie Abb. 4 zeigt.

Abschließend wird der Einfluss des Mindestlohns auf die Anwendung von Tarifverträgen in Betrieben des Bauhauptgewerbes untersucht, wobei verschiedene Wirkungen möglich sind. Zum einen ermöglicht die Mitgliedschaft in einem Arbeitgeberverband die Einflussnahme auf Verhandlungen der Lohnuntergrenzen. Zum anderen müssen die Mitarbeiter bei Bindung an den Branchentarifvertrag auch mindestens entsprechend ihrer Tarifstufe entlohnt werden, was die Lohnkosten im Betrieb steigen lässt. Die kausale Analyse mittels DvD-Schätzungen zeigt hier jedoch keinen Einfluss des Mindestlohns auf den Anteil tarifgebundener Unternehmen.

\section{Mindestlohn aus Sicht der Arbeitnehmer}

Die im Rahmen des Projekts durchgeführte Beschäftigtenbefragung ermöglicht ein aktuelles und repräsentatives Stimmungsbild der im Bauhauptgewerbe Beschäftigten hinsichtlich des Mindestlohns. 62 Prozent der Befragten in Westdeutschland wissen von einem Mindestlohn in ihrer Branche, 18 Prozent sind der Meinung, dass keine Lohnuntergrenze in ihrer Branche existiert, 20 Prozent wissen es nicht oder haben die Antwort verweigert. In Ostdeutschland dagegen geben 87 Prozent der Befragten an, dass ein Mindestlohn gültig ist und nur sieben Prozent verneinen dies. Unabhängig davon, ob ihre eigene Einschätzung richtig ist, deutet dieses Antwortmuster auf die größere Bedeutung des Mindestlohns im ostdeutschen Bauhauptgewerbe hin.
77 Prozent der Befragten in Westdeutschland und ca. 83 Prozent in Ostdeutschland erachten den Mindestlohn für das Bauhauptgewerbe als wichtig. Allerdings ist den meisten Arbeitern in den neuen Bundesländern der Mindestlohn zu niedrig (für 80 Prozent der Befragten), im Gegensatz zu 63 Prozent in den alten Bundesländern. Dieses Bild spiegelt sich auch insgesamt im Gerechtigkeitsempfinden gegenüber ihrem eigenen Stundenlohn wider. Für einen allgemeinen, gesetzlichen Mindestlohn sprechen sich 86 Prozent (Ostdeutschland) bzw. 79 Prozent (Westdeutschland) aus.

In Westdeutschland empfindet etwa die Hälfte der Beschäftigten ihren Stundenlohn als eher gerecht, in Ostdeutschland geben dies hingegen nur 17 Prozent an. 81 Prozent empfinden ihn dort als ungerecht, was wohl auf das insgesamt niedrigere Lohn- und auch Mindestlohnniveau sowie die geringere Lohnspreizung durch das schwache Tarifsystem in Ostdeutschland zurückzuführen sein dürfte, das ausgebildete und erfahrene Arbeiter kaum belohnt. Die Befragten in Westdeutschland würden ihren Lohn als gerecht empfinden, wenn er im Durchschnitt um 14 Prozent höher läge als ihr tatsächlicher. In Ostdeutschland wäre durchschnittlich eine 24-prozentige Lohnsteigerung für einen aus Sicht der aktuell unzufriedenen Befragten gerechten Lohn notwendig.

Sowohl in den alten als auch in den neuen Bundesländern stimmen rund die Hälfte der Befragten zumindest teilweise der Aussage zu, dass der Mindestlohn ihren Arbeitsplatz vor ausländischer Konkurrenz schützt. In Ostdeutschland ist die Zustimmung zu dieser Frage generell etwas stärker ausgeprägt als in Westdeutschland.

\section{Mindestlohn im Urteil der Branchenexperten}

Das Stimmungsbild unter den befragten Experten spiegelt wider, wie wichtig die Mindestlohnregelungen für das Bauhauptgewerbe im Allgemeinen sind. Am bedeutendsten sei hierbei die Höhe des Mindestlohns. Diese müsse hoch genug sein um eine beschäftigungssichernde Wirkung gegenüber entsendeten Arbeitnehmern zu entfalten und gering genug, um keine Arbeitsplätze einheimischer Arbeitnehmer zu gefährden. Seit seiner Einführung hätte er maßgeblich zu einem ,fairen“ Wettbewerb beigetragen, indem er bspw. eine Angleichung der Voraussetzungen bei der Auftragsvergabe geschaffen habe und eine wichtige Kalkulationsgrundlage bei der Unterbreitung von Angeboten biete. Insgesamt würde durch ihn sichergestellt, dass der Druck auf die Lohnkosten nur bis zum Mindestlohnniveau weitergegeben werden kann. Schutz biete er insbesondere gegenüber Unternehmen ohne Tarifbindung, organisierten Betrieben aus Nachbargewerken mit einem niedrigeren Mindestlohnniveau bzw. ohne Mindestlohn und vor ausländischer Niedriglohnkonkurrenz. 
Abb. 4 Preis- und Kostenentwicklung im Baugewerbe 1996-2010; Preisbereinigt; Kettenindex (1996=100). Quelle: Destatis, ELVIRA-Datenbank, eigene Berechnung

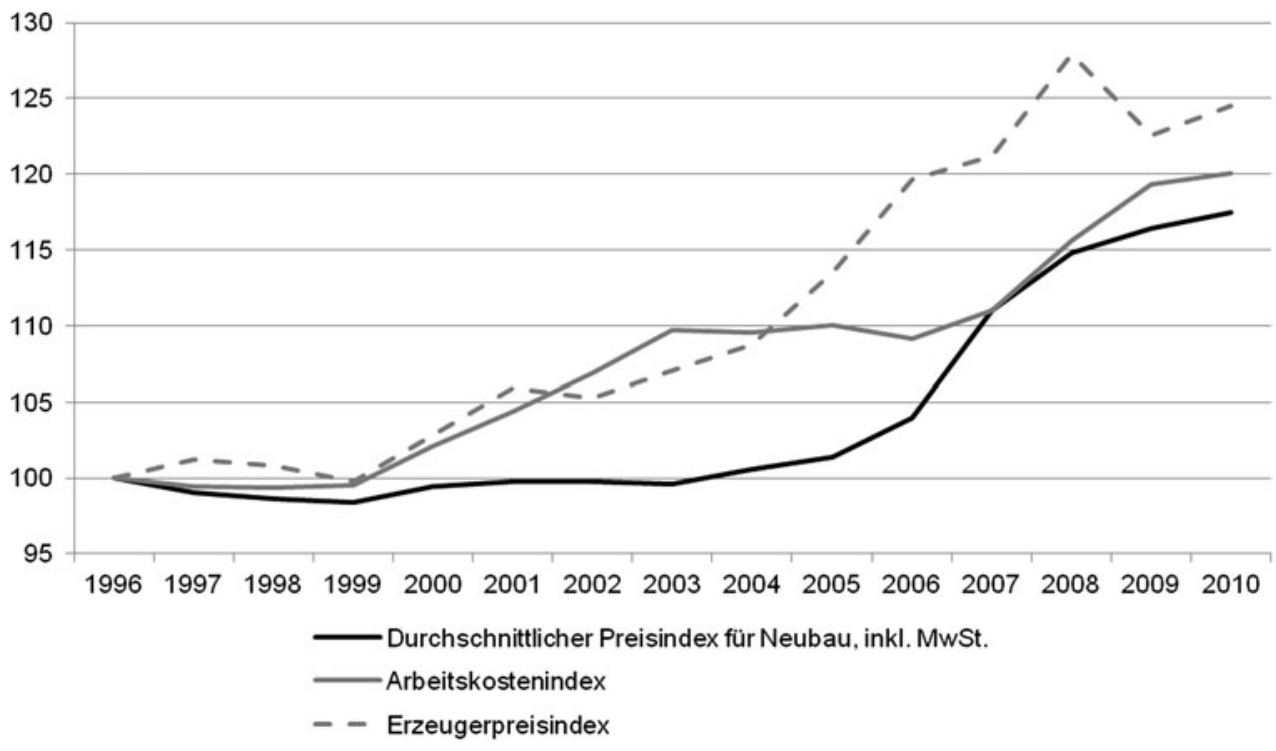

Ohne die Einführung des Mindestlohns wären die Beschäftigungsverluste während der Baurezession nach Meinung der befragten Experten noch deutlicher ausgefallen. Die Halbierung der deutschen Baubeschäftigung zwischen 1995 und 2005 sei weitestgehend auf den Umsatzrückgang nach Abflachen des ostdeutschen Baubooms zurückzuführen. Größere Entlassungswellen hätte der Mindestlohn hingegen nicht verursacht, sondern eher unterbunden. Mit einem durchschnittlichen Personalkostenanteil von 40 bis 60 Prozent an den Erstellungskosten hätte sich der ohnehin intensive Lohnwettbewerb weiter verschärft und ein Sinken des Durchschnittslohns unter das Mindestlohnniveau zur Folge gehabt. Darüber hinaus unterstreicht die Gewerkschaftsseite die hohe Bedeutung, die dem Mindestlohn bei der Wahrung des sozialen Friedens zukommt.

Bisweilen wird die Durchsetzbarkeit des Mindestlohns im Bauhauptgewerbe bemängelt, obgleich die Erhöhung der Kontrolldichte in den vergangenen Jahren als effizienzsteigernd wahrgenommen wird. Ein bekanntes Problem sei, dass bei der Auftragsvergabe häufig Angebote den Zuschlag erhielten, bei denen offenkundig nicht mit Lohnkosten auf Höhe bzw. oberhalb des Mindestlohnniveaus kalkuliert worden ist. In Hinblick auf das Bestehen von Schwarzarbeit, welche im Bauhauptgewerbe traditionell ein großes Problem sei, fällt das Urteil der Experten gemischt aus. Während einerseits die Steigerung der legalen Lohnkosten Schwarzarbeit attraktiver mache, wirke andererseits die verstärkte Kontrolldichte abschreckend. Zentral ist die unterschiedliche Bedeutung für die Lohnstruktur, die dem Mindestlohn zwischen den beiden Landesteilen beigemessen wird: Die Lohnuntergrenze wird in den alten Bundesländern überwiegend als Fundament für das Tarifgefüge angesehen, während sie in den neuen Bundesländern eher als ,direkte Lohnbasis“ fungiere. Den Gesprächspartnern der Verbände der Bauwirtschaft zufolge stütze der Mindestlohn in Westdeutschland die Tariflöhne und verhindere ein zu starkes Nachgeben am unteren Ende der Lohnverteilung. Da die einzelnen Mindestlohninterventionen überwiegend als Inflationsausgleich fungieren und die Tariflohnerhöhungen stärker ausfallen würden, bliebe das Tarifsystem stabil.

Aufgrund der in Ostdeutschland deutlich geringeren Tarifbindung hätte der Mindestlohn in den neuen Bundesländern zu einer Verringerung der Lohnspreizung bis hin zu einer Nivellierung auf das Mindestlohn-Niveau geführt. Nicht zuletzt auf Drängen der ostdeutschen Arbeitgeberverbände fiel im September 2009 der Mindestlohn II für Facharbeiter in Ostdeutschland weg. Aus Sicht der Gewerkschaftsvertreter hätte dies die beobachtete Lohnnivellierung in den neuen Bundesländern vorangetrieben, welche negative Auswirkungen auf den innerbetrieblichen Frieden habe. Aus Angst vor drohenden Arbeitsplatzverlusten würden höher qualifizierte ostdeutsche Arbeiter aber tendenziell den geringen Lohnabstand akzeptieren. In der jüngsten Entwicklung sei jedoch zu beobachten, dass ostdeutsche Baubetriebe Lohnzulagen oder anderweitige „Sonderzulagen“ für gute Fachkräfte bezahlen, vor allem um einer Abwanderung in die alten Bundesländer entgegen zu wirken. Als problematisch werden in diesem Zusammenhang insbesondere ein zunehmender Mangel an Fachkräften einerseits und ein Fernbleiben ausländischer Entsendearbeitnehmer andererseits gesehen.

Hinsichtlich der Wirkungsweisen des Mindestlohns auf die Entwicklung von Entsendungen besteht Uneinigkeit. Aus Sicht der Bauindustrie sowie des Baugewerbes habe dessen Einführung zumindest Anreize für den Einsatz von Subunternehmen aus Niedriglohnländern genommen. In diesem Zusammenhang wurde angemerkt, dass der Mindestlohn zu einer Verringerung der Lohndifferenz zwischen den Ländern beigetragen habe. Ihr vermehrter Einsatz sei 
lediglich noch auf westdeutschen Großbaustellen zu beobachten.

Weiterhin sei die Nachfrage nach qualifizierten Arbeitern durch den Mindestlohn gestiegen. Die Beschäftigung von Bauhelfern würde so gut wie nicht mehr beobachtet. Die im Bauhauptgewerbe generell geringe Bedeutung von Weiterbildungsmaßnahmen sei durch den Mindestlohn nicht negativ beeinflusst worden.

In Hinblick auf die zukünftige Entwicklung tendieren in erster Linie die Arbeitgeberverbände hin zu einem einheitlichen Mindestlohn von relativ geringer Höhe. Grundsätzlich müsse auch die Durchsetzbarkeit weiter erhöht werden, indem bspw. der Auftraggeber stärker zur Prüfung der Einhaltung gesetzlicher Regelungen verpflichtet wird, die Möglichkeiten zur Haftbarmachung des Generalunternehmens gegenüber Verstößen eines Subunternehmens weiter erhöht werden und Angebote bereits während des Ausschreibungsverfahrens auf Plausibilität geprüft werden können. Ein weiterer Missstand sei, dass eine Entlohnung unterhalb des Mindestlohns aus Angst vor einem Arbeitsplatzverlust kaum gemeldet würde. Dieses Problem bestünde insbesondere bei entsandten Arbeitnehmern, welche widrige Arbeitsbedingungen und eine geringe Entlohnung tendenziell akzeptierten, soweit sie sich ihrer Rechte überhaupt vollends bewusst seien.

\section{Fazit}

Im Bauhauptgewerbe wurde 1997 als erster Branche in Deutschland ein Mindestlohn eingeführt. Als Hauptgrund für die Einführung des Mindestlohns im Bauhauptgewerbe werden protektionistische Maßnahmen gegen die Zunahme der Entsendungen von Arbeitnehmern aus dem Ausland nach Deutschland angeführt. Die Verabschiedung des Gesetzes über zwingende Arbeitsbedingungen bei grenzüberschreitenden Dienstleistungen (AEntG) im Jahr 1996 ermöglicht eine Geltendmachung der für allgemeinverbindlich erklärten Mindestarbeitsbedingungen und damit der Mindestlöhne auch für aus dem Ausland entsandte Arbeitnehmer.

Zentralen Bestandteil dieses Artikels bilden die kausalanalytischen Untersuchungen der Mindestlohnwirkungen auf Löhne und Beschäftigung im Bauhauptgewerbe. Darüber hinaus wurden weiterführende Aspekte wie die Auswirkungen auf den Arbeitnehmerschutz und die Wettbewerbssituation betroffener Betriebe untersucht. Um tatsächliche Effekte der Mindestlohnregelung von weiteren Einflüssen, wie z.B. dem Konjunkturverlauf, abzugrenzen, wurde auf den Differenz-von-Differenzen Ansatz zurückgegriffen.

Qualitative Befunde deuten darauf hin, dass dem Mindestlohn in den neuen Bundesländern eine größere Bedeutung zukommt. Aussagen aus den Expertengesprächen unterstreichen, dass der Mindestlohn in den neuen Bundesländern als Fixpunkt fungiert, an dem sich die Tarifpartner orientieren, während er in den alten Bundesländern keinen Einfluss auf das Tarifsystem hat.

Die anschließenden Lohnanalysen zeigen, dass die Betroffenheit vom Mindestlohn in den neuen Bundesländern zum Zeitpunkt seiner Einführung deutlich höher war als in den alten Bundesländern. In den Jahren nach der Mindestlohneinführung verdichten sich die Stundenlöhne ostdeutscher Bauarbeiter an den jeweiligen Mindestlohnniveaus, während sich die Lohnspreizung in Westdeutschland kaum verändert. Die Kausalanalysen weisen auf positive Lohnwachstumseffekte der Mindestlohneinführung sowohl in West- als auch in Ostdeutschland hin, welche sich sowohl auf individueller als auch auf betrieblicher Ebene zeigen.

Die Einführung des Mindestlohns lässt in der Kausalanalyse keine messbaren Auswirkungen auf das Beschäftigungsniveau erkennen. Auf die Entlassungs- und Einstellungswahrscheinlichkeiten wirkt sich der Mindestlohn kaum aus, mit Ausnahme eines negativen Effekts der Mindestlohneinführung auf die Einstellungswahrscheinlichkeit in Ostdeutschland zeigen sich hier keine Effekte. Die Arbeitgeber im Bauhauptgewerbe weisen im Vergleich zu anderen Branchen eine relativ hohe Marktmacht auf, so dass Beschäftigungsverluste auch nicht zwangsläufig zu erwarten waren. Die vermuteten indirekten Wirkungen des Mindestlohns auf Weiterbildungen, befristete Beschäftigung oder gemeldeten Überstunden können ebenfalls nicht nachgewiesen werden.

Letztendlich ist einschränkend anzumerken, dass bestimmte Wirkungsweisen des Mindestlohns auf Aspekte wie Entsendungen, Schwarzarbeit, Arbeitszufriedenheit und Preisentwicklung nicht kausalanalytisch untersucht werden können. Insbesondere in Hinblick auf mögliche Beschäftigungseffekte auf Entsendearbeitnehmer können keine abschließenden Aussagen getroffen werden. Des Weiteren beschränkt sich die Analyse aufgrund methodischer Überlegungen auf die kurzfristigen Auswirkungen der Mindestlohneinführung.

Abschließend sollte beachtet werden, dass sich das Bauhauptgewerbe aufgrund einzigartiger Produktionsbedingungen deutlich von anderen Branchen unterscheidet, was sich in spezifischen tariflichen Rahmenbedingungen und einer bauspezifischen Arbeitsmarktpolitik widerspiegelt. Daher wird die Übertragbarkeit der Ergebnisse für das Bauhauptgewerbe auf andere Wirtschaftszweige in Deutschland auch in Bezug auf die Einführung eines allgemeinen gesetzlichen Mindestlohns als relativ gering eingeschätzt.

\section{Kurzfassung}

Im Bauhauptgewerbe wurde zum 01. Januar 1997 als erster Branche in Deutschland ein Mindestlohn eingeführt. Der 
vorliegende Artikel fasst die Ergebnisse dieser Einführung im Rahmen der vom Bundesministerium für Arbeit und Soziales (BMAS) in Auftrag gegebenen Studie zur „Evaluation bestehender gesetzlicher Mindestlohnregelungen - Branche: Bauhauptgewerbe" zusammen.

Als Hauptgrund für die Einführung des Mindestlohns im Bauhauptgewerbe gelten protektionistische Maßnahmen gegen die Zunahme an Entsendungen von bei ausländischen Unternehmen beschäftigten Arbeitnehmern nach Deutschland. Das „Gesetz über zwingende Arbeitsbedingungen bei grenzüberschreitenden Dienstleistungen“ (AEntG) wurde 1996 verabschiedet und ist seit dem 01. Januar 1997 in Kraft. Seitdem gelten im Bauhauptgewerbe für allgemeinverbindlich erklärte Mindestarbeitsbedingungen, und damit auch der Mindestlohn, sowohl für Beschäftigte deutscher Unternehmen, als auch für Arbeitnehmer, die aus dem Ausland nach Deutschland entsandt wurden.

Das Hauptaugenmerk der im Artikel vorgestellten Analysen liegt auf den kausalanalytischen Untersuchungen der Mindestlohnwirkungen auf Löhne und Beschäftigung im Bauhauptgewerbe. Zusätzlich wird auf weitere Aspekte, wie z.B. den Arbeitnehmerschutz und die Wettbewerbssituation betroffener Betriebe, eingegangen. Die Analysen wurden auf verschiedenen Beobachtungsebenen durchgeführt, um ein möglichst umfassendes Bild zu zeichnen. So erfolgte die Untersuchung auf individueller, sektoraler und regionaler Ebene. Um mögliche Wirkungen der Mindestlohneinführung von weiteren Einflüssen, wie beispielsweise dem Konjunkturverlauf, abgrenzen zu können, wurde auf den Differenz-von-Differenzen Ansatz zurückgegriffen, der auf einem Vergleich zwischen einer Treatment- und einer Kontrollgruppe beruht. Bezüglich der Treatment- und Kontrollgruppeneinteilung wurden zwei alternative Strategien verfolgt. Zum einen wurden direkt vom Mindestlohn betroffene Beschäftigte bzw. Betriebe mit nicht betroffenen Beschäftigten bzw. Betrieben innerhalb des Bauhauptgewerbes verglichen. Zum anderen erfolgte der Vergleich zwischen Beschäftigten bzw. Betrieben im Bauhauptgewerbe und Beschäftigten bzw. Betrieben in ausgewählten Kontrollbranchen.

Als Datenbasis diente ein eigens für das Projekt erstellter Datensatz, welcher das gesamte Bauhauptgewerbe umfasst. Hierfür wurden administrative Daten der Bundesagentur für Arbeit und des Instituts für Arbeitsmarkt- und Berufsforschung (IAB) sowie das IAB-Betriebspanel und Informationen aus dem Mikrozensus zusammengeführt. Zusätzlich wurden zu Jahresbeginn 2011 über 1500 Beschäftigte des Bauhauptgewerbes telefonisch u.a. zum Mindestlohn, zu ihren Arbeitsbedingungen sowie zu Aspekten der Schwarzarbeit interviewt. Diese Befragungsdaten wurden ebenfalls hinzu gespielt und ausgewertet. Für deskriptive Analysen konnte darüber hinaus auf Daten aus dem Meldeverfahren der SOKA-BAU sowie der statistischen Datenbank des
Hauptverbands der Deutschen Bauindustrie zurückgegriffen werden.

Um spezifische Hintergrundinformationen aus unterschiedlichen Perspektiven über die Besonderheiten der Baubranche, v.a. in Bezug auf die Mindestlohnregelungen und Bewertungen der Akteure, zu erhalten, wurden neben den quantitativen Analysen Expertengespräche mit den Hauptakteuren im Bauhauptgewerbe geführt.

Die quantitativen Lohnanalysen zeigen, dass die Betroffenheit vom Mindestlohn in den neuen Bundesländern zum Zeitpunkt seiner Einführung deutlich höher war als in den alten Bundesländern. In den Jahren nach der Mindestlohneinführung verdichten sich die Stundenlöhne ostdeutscher Bauarbeiter an den jeweiligen Mindestlohnniveaus, während sich die Lohnspreizung in Westdeutschland kaum verändert. Die Kausalanalysen weisen auf positive Lohnwachstumseffekte der Mindestlohneinführung sowohl in West- als auch in Ostdeutschland hin, welche sich sowohl auf individueller als auch auf betrieblicher Ebene zeigen.

Die qualitativen Befunde bestätigen das Ergebnis, dass dem Mindestlohn im ostdeutschen Bauhauptgewerbe eine deutlich größere Bedeutung zukommt als in den alten Bundesländern. Aussagen aus den Expertengesprächen unterstreichen zudem, dass der Mindestlohn in den neuen Bundesländern als Fixpunkt fungiert, an dem sich die Tarifpartner orientieren, während er in den alten Bundesländern relativ wenig Einfluss auf das Tarifsystem hat.

Die Einführung des Mindestlohns lässt in der Kausalanalyse keine messbaren Auswirkungen auf das Beschäftigungsniveau erkennen. Auf die Entlassungs- und Einstellungswahrscheinlichkeiten wirkt sich der Mindestlohn kaum aus, mit Ausnahme eines negativen Effekts der Mindestlohneinführung auf die Einstellungswahrscheinlichkeit in Ostdeutschland zeigen sich hier keine weiteren Effekte. Die Arbeitgeber im Bauhauptgewerbe weisen im Vergleich zu anderen Branchen eine relativ hohe Marktmacht auf, so dass Beschäftigungsverluste auch nicht zwangsläufig zu erwarten sind. Mögliche indirekte Wirkungen des Mindestlohns auf Weiterbildungen, befristete Beschäftigung oder gemeldete Überstunden können ebenfalls nicht nachgewiesen werden. Auch hinsichtlich der Wettbewerbsfähigkeit zeigen sich keine kausalen Effekte der Mindestlohneinführung. Auswertungen der Beschäftigtenbefragung sowie der Expertengespräche zeigen, dass sowohl Arbeitgeber als auch Arbeitnehmer die Mindestlohnregelungen für sehr wichtig halten. Verbesserungspotenzial biete sich allerdings hinsichtlich der Kontrolle der Mindestlohneinhaltung. Als problematisch wird teilweise auch die fehlende Lohnspreizung in Ostdeutschland eingeschätzt.

Einschränkend anzumerken ist, dass bestimmte Wirkungsweisen des Mindestlohns auf Aspekte wie Entsendungen, Schwarzarbeit, Arbeitszufriedenheit und Preisentwicklung nicht kausalanalytisch untersucht werden können. 
Insbesondere in Hinblick auf mögliche Beschäftigungseffekte auf entsandte Arbeitnehmer können keine abschlieBenden Aussagen getroffen werden. Des Weiteren beschränkt sich die Analyse aufgrund methodischer Überlegungen auf die kurzfristigen Auswirkungen der Mindestlohneinführung.

Abschließend ist darauf hinzuweisen, dass sich das Bauhauptgewerbe aufgrund der Produktionsbedingungen deutlich von anderen Branchen unterscheidet, was sich in spezifischen tariflichen Rahmenbedingungen und einer bauspezifischen Arbeitsmarktpolitik widerspiegelt. Eine mögliche Übertragbarkeit der Ergebnisse auf andere Wirtschaftszweige und auch in Bezug auf die Einführung eines allgemeinen gesetzlichen Mindestlohns wird als eher gering eingeschätzt.

\section{Executive summary}

The first sectoral minimum wage in Germany was introduced in the main construction sector on January 1st, 1997. This article summarizes the results of this introduction which was analyzed in detail within the scope of the study "Evaluation bestehender gesetzlicher Mindestlohnregelungen - Branche: Bauhauptgewerbe" commissioned by the German Federal Ministry of Labour and Social Affairs (BMAS). One of the main reasons for the introduction of a minimum wage in the construction sector was to protect workers against foreign competition by posting foreign workers to Germany. By passing the Posting of Workers Law in 1996, compulsory working conditions (and thus minimum wages) apply for German employees as well as for posted workers. The main focus of the article is on the analyses of the minimum wage impacts on wages and employment in the main construction sector. Additional aspects comprise worker protection and competition. The analyses are conducted on different observational levels (individual, sectoral and regional) in order to obtain a comprehensive picture. We use a difference-in-differences approach classifying observations into treatment and control groups in order to isolate possible impacts of the minimum wage introduction from other influences like the business cycle. We follow two alternative strategies to assign the treatment and control group. First, directly affected individuals or establishments are compared to those not directly affected within the main construction sector. Second, we conduct a comparison between individuals or establishments in the main construction sector and selected control industries.

We use a data set for the main construction sector specially assembled for this project. For this purpose, we merge administrative data of the German Federal Employment Agency (BA) and the Institute for Employment Research (IAB) as well as the IAB Establishment Panel and information from the German Micro Census. Over 1500 employees of the main construction sector were interviewed by phone at the beginning of 2011 regarding the minimum wage, their working conditions and issues related to illegal employment. This survey is also analyzed. Moreover, we draw on data of the notification procedure of the social security fund for construction (SOKA-BAU) as well as the statistical data base of the construction industry association (Hauptverband der Deutschen Bauindustrie). In addition to quantitative analyses, information from expert discussions with main actors in the construction sector are used to obtain specific background information from different perspectives on characteristics of the construction industry, especially with regard to the minimum wage regulations. Qualitative findings point to the fact that the minimum wage has a significantly higher importance in the East German main construction sector compared to West Germany.

The wage analysis shows that the degree of being affected by the minimum wage was significantly higher in East Germany at the time of the introduction as compared to West Germany. The hourly wages of East German workers concentrate at the corresponding minimum wage level in the years after the minimum wage introduction. The wage dispersion in West Germany hardly changes.

The causal analyses point to positive wage growth effects of the minimum wage introduction in East as well as in West Germany. They are visible at the individual and the establishment level. The results from the qualitative analysis confirm the finding from the quantitative analysis that the minimum wage plays a much more important role in East Germany than in West Germany. In particular, statements from the expert talks emphasize that the minimum wage serves as orientation point for the social partners in East Germany. In West Germany, the influence of the minimum wage on the collective bargaining system is relatively low.

The minimum wage introduction does not reveal measurable impacts on the level of employment. The minimum wage barely influenced hiring and separation probabilities except for a negative effect on the hiring probability in East Germany.

Employment losses may not necessarily be expected as employers in the main construction sector have relatively high market power compared to other sectors. Additionally, we cannot detect indirect impacts of the minimum wage on training, temporary employment and reported overtime. Neither did the analyses reveal causal effects of the minimum wage introduction on competitiveness. Results from the employee survey as well as the expert discussions highlight that employers as well as employees consider the minimum wage regulations as important. Room for improvement concerns supervision of compliance. They also partly assess the lack of wage dispersion in East Germany as a problem. 
Certain impacts of the minimum wage could not be analyzed in a causal way like posting workers, illegal employment, job satisfaction, and development of prices. We cannot make conclusive statements about possible employment effects for posted workers from other countries. Furthermore, the analysis is restricted to short-term effects due to methodological considerations.

Finally, it should be noted that the main construction sector differs from other sectors due to special conditions of production. This is reflected by specific collectively agreed conditions and a construction specific labor market policy. Transferring the results to other sectors or using them in the context of an introduction of a generally binding minimum wage thus seems inadvisable.

\section{Literatur}

Angrist, J., Krueger, A.: Empirical strategies in labor economics. In: Ashenfelter, O., Card, D. (Hrsg.) Handbook of Labor Economics, vol. 3A, pp. 1278-1366. North Holland, Amsterdam (1999)

Apel, H.: Das methodische Konzept der Fallstudien des ISG. ISG Working Paper No. 6 (2009)

Bauer, H.: Baubetrieb, 2. Aufl. Springer, Berlin (1995)

Bosch, G., Weinkopf, C., Worthmann, G.: Die Fragilität des Tarifsystems - Einhaltung von Entgeltstandards und Mindestlöhnen am Beispiel des Bauhauptgewerbes. Forschung aus der HansBöckler-Stiftung, edition sigma, Berlin (2011)

Bosch, G., Worthmann, G., Zühlke-Robinet, K.: Das deutsche Baugewerbe im Europäischen Wettbewerb. In: Sadowski, D., Walwei, U. (Hrsg.) Die ökonomische Analyse des Arbeitsrechts: IAB-Kontaktseminar vom 12.-16.11.2001 im Institut für Arbeitsrecht und Arbeitsbeziehungen in der Europäischen Gemeinschaft (IAAEG) der Universität Trier. Beiträge zur Arbeitsmarkt- und Berufsforschung, vol. 259, S. 107-143 (2002)

Bosch, G., Zühlke-Robinet, K.: Der Bauarbeitsmarkt - Soziologie und Ökonomie einer Branche. Campus Verlag, Frankfurt (2000)

Cremers, J.: Auf der suche nach billigen Arbeitskräften in Europa - Lebens- und Arbeitsbedingungen entsandter Arbeitnehmer. In: European Institute for Construction Labour Research, CLR Studies, S. 6 (2011)

Destatis (Hrsg.): Genesis-Online Datenbank, Volkswirtschaftliche Gesamtrechnungen des Bundes. Statistisches Bundesamt, Wiesbaden (2011)

Dolton, P., Bondibene, C., Wadsworth, J.: Employment, inequality and the UK national minimum wage over the Medium-Term. Oxf. Bull. Econ. Stat. 74(1), 78-106 (2012)

Eckey, H.-F., Schwengler, B., Türck, M.: Vergleich von deutschen Arbeitsmarktregionen. IAB Discussion Paper No. 3/2007 (2007)

Fischer, G., Janik, F., Müller, D., Schmucker, A.: The IAB establishment panel - from sample to survey to projection. FDZ Methodenreport Nr. 01/2008. Nürnberg (2008)

Frings, H.: The employment effect of industry-specific, collectivelybargained minimum wages. Ruhr Economic Papers 348, RWI (2012)

Hethey, T., Schmieder, J.: Using worker flows in the analysis of establishment turnover - evidence from German Administrative data. FDZ Methodenreport 06/2010 (2010)

Hethey-Maier, T., Seth, S.: Das Betriebs-Historik-Panel (BHP) 19752008. Handbuch Version 1.0.2. FDZ Datenreport 04/2010 Nürnberg (2010)

Hirsch, B.: Monopsonistic Labour Markets and the Gender Pay Gap: Theory and Empirical Evidence. Springer, Heidelberg (2010)
Hochstadt, S.: Die Zukunft der Qualifikation in der Bauwirtschaft Innere und äußere Momente des Strukturwandels. Dissertation, Universität Osnabrück (2002)

Institut für Arbeitsmarkt- und Berufsforschung/Rheinisch-Westfälisches Institut für Wirtschaftsforschung/Institut für Sozialforschung und Gesellschaftspolitik [IAB/RWI/ISG]: Evaluation bestehender gesetzlicher Mindestlohnregelungen - Branche: Bauhauptgewerbe. Nürnberg/Essen/Köln (2011)

IW Consult: Analyse der volkswirtschaftlichen Bedeutung der Wertschöpfungskette Bau - Endbericht. Institut der deutschen Wirtschaft Köln Consult GmbH, Köln (2008)

Kahmann, M.: The posting of workers in the German construction industry: responses and problems of trade union actions. Eur. Rev. Labour Res. 12(2), 183-196 (2006)

Kofner, S.: Zukunftsperspektiven der deutschen Bauwirtschaft. Wirtschaftspolitische Diskurse des Forschungsinstitutes der FriedrichEbert-Stiftung 111, Bonn (1998)

König, M., Möller, J.: Impacts of minimum wages - a microdata analysis for the German construction sector. Int. J. Manpow. 30(7), 716-741 (2009)

Lechner, M.: The estimation of causal effects by Difference-inDifference methods. University of St. Gallen Department of Economics working paper series 2010-28, revised Oct 2011 (2010)

Manning, A.: Monopsony in Motion. Princeton University Press, Princeton (2003)

Oberschachtsiek, D., Scioch, P., Seysen, C., Heining, J.: In: Stichprobe der Integrierten Erwerbsbiografien IEBS. Handbuch für die IEBS in der Fassung 2008. FDZ Datenreport, 03/2009, Nürnberg, (2009)

Platzer, L., Röschert, B.: Tarifverträge Arbeitsrecht Bau 2005, VOBVerlag. Ernst Vögel, Stamsried (2005)

Rattenhuber, P.: Building the minimum wage. Germany's first sectoral minimum wage and its impact on wages in the construction industry. DIW Discussion papers Nr. 1111, Berlin (2011)

Sachverständigenrat zur Begutachtung der gesamtwirtschaftlichen Entwicklung (2011)

Schneider, A., Thoenes, H.J., Trageser, H.: Die Deutsche Bauwirtschaft - Wachstum und Strukturwandel Seit 1960. Veröffentlichungen des HWWA-Instituts für Wirtschaftsforschung. Verlag Weltarchiv GmbH, Hamburg (1982)

Unger, B., van Waarden, F.: A comparison of the construction industry in Europe - Characteristics, governance, performance and future perspectives. Arbeitshefte Wirtschaftsuniversität Wien Reihe Volkswirtschaft, Working paper No. 18 (1993)

Worthmann, G.: Der Bauarbeitsmarkt unter Veränderungsdruck. Kontrolldefizit in folge der Transnationalisierung? In: Institut Arbeit und Technik: Jahrbuch 1997/98, Gelsenkirchen, S. 70-85 (1997)

Worthmann, G., Zühlke-Robinet, K.: Neue Arbeitsmigration im Baugewerbe und ihre Regulierung - das Arbeitnehmer-Entsendegesetz als Instrument zur Re-Regulierung des Bauarbeitsmarktes. In: Hunger, U., Santel, B. (Hrsg.) Migration im Wettbewerbsstaat. Leske + Budrich: Opladen, S. 91-118 (2003)

Dr. Helmut Apel Studium der Sozialwissenschaften und der Soziologie an der Universität Würzburg und der Universität Marburg, 1988 Abschluss als Diplom-Soziologe. Von 1988-1992 wissenschaftlicher Mitarbeiter an der Universität Marburg und Siegen. 1992 Promotion zum Dr. Phil. am Fachbereich Gesellschaftswissenschaften und Philosophie der Universität Marburg. Anschließend Tätigkeit als Studienleiter im Emnid-Institut bis 1997. Seit 1998 wissenschaftlicher Mitarbeiter des Instituts für Sozialforschung und Gesellschaftspolitik (ISG), Köln.

Forschungsfelder: Arbeitsmarktforschung, Evaluation arbeitsmarktpolitischer Programme, ,atypische“ Beschäftigung, europäische Forschungsförderung, Innovationsforschung, Sozialberichterstattung. 
Dr. Ronald Bachmann ist stellvertretender Leiter des Kompetenzbereichs „Arbeitsmärkte, Bildung, Bevölkerung“ am Rheinisch-Westfälischen Institut für Wirtschaftsforschung. Er studierte Volkswirtschaft an der University of Cambridge, England, und an der London School of Economics. Danach promovierte er an der Humboldt-Universität zu Berlin. Seine Forschungsinteressen liegen in der angewandten Arbeitsmarktökonomik, insbesondere der Analyse von Arbeitsmarktdynamiken und von verschiedenen Maßnahmen der Arbeitsmarktpolitik. Er war zudem in vielfältigen Evaluationsprojekten, z.B. im Auftrag von verschiedenen Bundesministerien und der Europäischen Kommission, tätig.

Stefan Bender studierte Soziologie und Statistik in Mannheim. Sein Studium schloss er 1990 als Diplom-Soziologe ab. Von 1990-1992 war er wissenschaftlicher Mitarbeiter an der Universität Mannheim. Seit 1992 ist er als wissenschaftlicher Mitarbeiter im IAB und seit 2005 leitet er das Forschungsdatenzentrum der Bundesagentur für Arbeit im IAB. Er ist Mitglied beim Rat für Sozial- und Wirtschaftsdaten (RatSWD). Seine Forschung beschäftigt sich mit dem Verknüpfen/Linken und der Qualität von Daten, dem Datenschutz und dem Datenzugang. Ein weiterer Schwerpunkt sind seine Arbeiten in der Arbeitsmarktökonomie, wo er zuletzt zu den Themen Arbeitslosigkeit, Lohnverlust, Weiterbildung und Innovation forschte und in einschlägigen Fachzeitschriften publizierte.

Philipp vom Berge studierte Volkswirtschaftslehre an der Universität Regensburg und schloss sein Studium 2007 als Diplom-Volkswirt ab. Bis 2012 war er wissenschaftlicher Mitarbeiter am Lehrstuhl für Volkswirtschaftslehre an der Universität Regensburg. Seit 2011 ist er wissenschaftlicher Mitarbeiter im Forschungsdatenzentrum der Bundesagentur für Arbeit im Institut für Arbeitsmarkt- und Berufsforschung (IAB). Seine Forschungsinteressen liegen in den Bereichen Regional- und Arbeitsmarktökonomik.

Dr. Michael Fertig studierte Volkswirtschaftslehre an der Universität Heidelberg und wurde dort promoviert. Von 2002 bis 2006 war er als Forschungskoordinator am Rheinisch-Westfälischen Institut für Wirtschaftsforschung beschäftigt. Seit 2007 ist er geschäftsführender Gesellschafter des Instituts für Sozialforschung und Gesellschaftspolitik. $\mathrm{Zu}$ seinen Forschungsschwerpunkten zählen Arbeitsmarkt-, Bildungsund Bevölkerungsökonomik. In diesen Bereichen hat er in zahlreichen Fachzeitschriften publiziert und an einer Vielzahl an Gutachten mitgewirkt.

Hanna Frings ist seit August 2009 als Wissenschaftlerin im Kompetenzbereich „Arbeitsmärkte, Bildung, Bevölkerung“ des RheinischWestfälischen Instituts für Wirtschaftsforschung (RWI) tätig. Sie studierte Volkswirtschaftslehre an der Universität Maastricht (Niederlande) mit einem Schwerpunkt auf Wirtschaftspolitik und befasst sich im Rahmen ihrer Promotion mit den Auswirkungen von Mindestlöhnen. Frau Frings Forschungsinteressen liegen in der angewandten Ökonometrie sowie in den Institutionen des Arbeitsmarkts. Während ihrer Tätigkeit am RWI hat sie bei verschiedenen Projekten im Auftrag der Europäischen Kommission und des Bundesministerium für Arbeit und Soziales mitgearbeitet.

Marion König Studium der Volkswirtschaftslehre an der Universität Regensburg und Universidad de Barcelona (Spanien). 2006 Abschluss als Diplom-Volkswirtin. Von 2006 bis 2010 wissenschaftliche Mitarbeiterin an der Universität Regensburg. Seit 2008 wissenschaftliche Mitarbeiterin am Institut für Arbeitsmarkt- und Berufsforschung in der Forschungsgruppe des Direktors sowie seit 2010 im Forschungsdatenzentrum der BA im IAB. Forschungsfelder: Arbeitsmarktökonomie, insbesondere Wirkungen von Mindestlöhnen und geschlechtsspezifische Lohnungleichheit im regionalen Vergleich.

Prof. Dr. Dr. h.c. Joachim Möller Studium der Philosophie und Volkswirtschaftslehre an den Universitäten Tübingen, Straßburg und Konstanz, 1978 Abschluss als Diplom-Volkswirt. 1981 Promotion zum Dr. rer. soc. und 1990 Habilitation an der Universität Konstanz. Seit 1991 Professor für Volkswirtschaftslehre an der Universität Regensburg. Von 2005-2007 Direktor des Osteuropa-Instituts München. Seit dem 01.10.2007 Direktor des Instituts für Arbeitsmarkt- und Berufsforschung der Bundesagentur für Arbeit. Forschungsfelder: Arbeitsmarktökonomie, Regionalökonomie, Empirische Makroökonomie, Angewandte Ökonometrie.

Dr. Alfredo Paloyo ist wissenschaftlicher Mitarbeiter im Kompetenzbereich „Arbeitsmärkte, Bildung, Bevölkerung“ am RWI in Essen. Er absolvierte seine Promotion an der Ruhr Graduate School in Economics. Zuvor erhielt er einen Master of Laws der Erasmus-Universität in Rotterdam, der Universität Hamburg sowie der Universität Bologna. Seine Forschungsschwerpunkte liegen derzeit in den Bereichen Verteidigungs-, Arbeitsmarkt-, Gesundheits- und Regionalökonomie. Seine Arbeiten wurden u.a. kürzlich im European Economic Review und Defence and Peace Economics veröffentlicht.

Dr. Sandra Schaffner studierte an der Technischen Universität Darmstadt Wirtschaftsingenieurwesen mit technischer Fachrichtung Elektrotechnik (Nachrichten- und Kommunikationstechnik). Sie schloss 2004 ihr Studium als Diplom-Wirtschaftsingenieurin ab und ist seit April 2005 als wissenschaftliche Mitarbeiterin im RWI in Essen beschäftigt. Seit Januar 2012 leitet sie das Forschungsdatenzentrum Ruhr am RWI. Während ihrer Tätigkeit am RWI wurde sie bei Prof. Christoph M. Schmidt an der Ruhr-Universität Bochum promoviert. Ihr Forschungsschwerpunkt ist die empirische Arbeitsmarktökonometrie insbesondere kompensatorische Lohndifferentiale, Arbeitsmarktdynamik und Evaluation von Politikmaßnahmen.

Dr. Marcus Tamm ist Leiter des Kompetenzbereichs „Arbeitsmärkte, Bildung, Bevölkerung “ am RWI in Essen. Er studierte Volkswirtschaftslehre an den Universitäten Würzburg, Salamanca und Heidelberg. 2007 beendete er seine Promotion an der Ruhr-Universität Bochum. Seine Arbeitsschwerpunkte liegen im Bereich Familien- und Bildungsökonomik und in der Evaluation von Arbeitsmarktreformen.

Matthias Umkehrer studierte Volkswirtschaftslehre an der Universität Regensburg und schloss sein Studium im November 2010 als Master of Science (M.Sc.) ab. Seit Januar 2011 ist er wissenschaftlicher Mitarbeiter im Forschungsdatenzentrum der Bundesagentur für Arbeit im Institut für Arbeitsmarkt- und Berufsforschung (IAB) und seit November 2011 ist er Stipendiat im Graduiertenprogramm des IAB. Seine akademischen Interessen umfassen Arbeitsmarktökonomie und Mikroökonometrie mit besonderer Ausrichtung auf die Bereiche frühe Erwerbsverläufe und Jugendarbeitslosigkeit. In seiner Dissertation untersucht er Wirkungen von frühen Erfahrungen auf dem Arbeitsmarkt auf zukünftige Arbeitsmarktergebnisse.

Stefanie Wolter studierte an der Technischen Universität in Chemnitz European Studies und absolvierte währenddessen ein Auslandssemester an der Wirtschaftsuniversität Wroclaw. Anschließend schloss sie den Masterstudiengang International Economics an der Universität Regensburg als Master of Science ab. Seit Juli 2011 ist die wissenschaftliche Mitarbeiterin im Forschungsdatenzentrum der Bundesagentur für Arbeit im Institut für Arbeitsmarkt- und Berufsforschung (IAB). Ihr Forschungsinteresse gilt Erfolgsfaktoren von Betrieben und Qualität von Beschäftigung. 